\title{
Dynamics and Structure of Room Temperature Ionic Liquids
}

\author{
Michael D. Fayer \\ Department of Chemistry \\ Stanford University, Stanford, CA 94305-5080 \\ fayer@stanford.edu
}

\begin{abstract}
Room temperature ionic liquids (RTIL) are intrinsically interesting because they simultaneously have properties that are similar to organic liquids and liquid salts. In addition, RTILs are increasingly being considered for and used in technological applications. RTILs are usually composed of an organic cation and an inorganic anion. The organic cation, such as imidazolium, has alkyl chains of various lengths. The disorder in the liquid produced by the presence of the alkyl groups lowers the temperature for crystallization below room temperature and can also result in supercooling and glass formation rather than crystallization. The presence of the alkyl moieties also results in a segregation of the liquid into ionic and organic regions. In this article, experiments are presented that address the relationship between RTIL dynamics and structure. Time resolved fluorescence anisotropy measurements were employed to study the local environments in the organic and ionic regions of RTILs using a nonpolar chromophore that locates in the organic regions and an ionic chromophore that locates in the ionic regions. In the alkyl regions, the in plane and out of plane orientational friction coefficients change in different manners as the alkyl chains get longer. Both friction coefficients converge toward those of a long chain length hydrocarbon as the RTIL chains increase in length, which demonstrates that for sufficiently long alkyl chains the RTIL organic regions have properties similar to a hydrocarbon. However, putting $\mathrm{Li}^{+}$in the ionic regions changes the friction coefficients in the alkyl regions, which


demonstrates that changes of the ion structural organization influences the organization of the alkyl chains. Optical heterodyne detected optical Kerr effect (OHD-OKE) experiments were used to examine the orientational relaxation dynamics of RTILs over times scales of a hundred femtoseconds to a hundred nanoseconds. Detailed temperature dependent studies in the liquid and supercooled state and analysis using schematic mode coupling theory (MCT) show that RTILs have bulk liquid orientational relaxation dynamics that are indistinguishable in their nature from common nonpolar organic liquids that supercool. This behavior of the RTILs occurs in spite of the segregation into ionic and organic regions. However, when small amounts of water are added to RTILs at room temperature, novel dynamics are observed for the RTILs with long alkyl chains that have not been observed in OHD-OKE experiments on organic liquids. The results are interpreted as water induced structure in the ionic regions that causes the long alkyl chains to organize and "lock up." The dynamical measurements indicate that this lock up is involved in the formation of RTIL gels that occur over a narrow range of water concentrations. 


\section{Introduction}

Over the last decade, room temperature ionic liquids (RTILs) have generated a great deal of interest because of their very low volatility, enormous variability, and good thermal stability. RTILs are salts with a melting point below $25^{\circ} \mathrm{C}$. They are often composed of an inorganic anion paired with an asymmetric organic cation that contains one or more pendant alkyl chains. The asymmetry of the cation frustrates crystallization, causing the salt's melting point to drop substantially compared to a common salt such as $\mathrm{NaCl}$. RTILs have applications in organic synthesis, electrochemistry, and separation processes.(1-5) They are also being investigated for a variety of other applications, such as us in batteries.(6)

For a number of years, the general consensus was that there was significant mesoscopic structure, with the long, hydrocarbon tails aggregating to form non-polar domains and with the cationic head groups and the anions forming tortuous ion channels that percolated through the pure liquid on relatively long length scales. This view was supported by a number of theoretical studies.(7-11) X-ray scattering experiments initially seemed to support such mesoscopic organization.(12-14) Within that framework, many experiments, including fluorescence,(15-17) optical Kerr effect,(18-22) and dielectric spectroscopies; $(21,23)$ NMR; $(24,25)$ and small-angle neutron scattering (SANS)(26) were interpreted by invoking the extended structure concept.

However, in 2010, H/D isotope labeled SANS experiments(27) and MD simulations $(28)$ called into question the interpretation of the x-ray data. The SANS results showed that the x-ray data could be explained by anisotropic solvation, in which the alkyl-cation is preferentially solvated on the alkyl tail side by other alkyl tails and on 
the imidazolium side by anions. Recent $\mathrm{x}$-ray scattering experiments on binary mixtures of RTILs and on RTILs with polar polyether tails were again interpreted as supporting the long range correlations.(29) Both mesoscopic structure and more local nanoscopic heterogeneity have been invoked in recent papers.(30-34) Detailed x-ray scattering experiments and theoretical analysis demonstrated that there is nanoscopic heterogeneity of the ionic and organic regions, but no extensive mesoscopic structuring.(30) A recent study of nanostructure using electronic excitation transfer experiments is consistent with the $\mathrm{x}$-ray results.(35)

While the question of mesoscopic heterogeneity may not be fully settled, there is no doubt that RTILs contain nanoscopic heterogeneity with hydrophobic regions of alkyl tails, and charge-ordered, hydrophilic head group regions that are distinct at least when the alkyl tails are sufficiently long, greater in length than ethyl. In the context of this heterogeneous liquid structure, highly selective solvation is possible.(36) The resulting diversity of local environments can have a direct impact on the dynamics of processes where solvation environment is important. The effect of environment has been observed in the context of a photoisomerization.(37) Anomalous reaction rates in RTILs have been attributed to this nanoscale structural heterogeneity. $(38,39)$ Dynamic heterogeneity could influence transport properties and availability of reagents and catalysts. One appeal of RTILs as a reaction medium is the breadth of molecules they can solvate because of the presence of multiple chemical environments. Dynamic heterogeneity may have a nontrivial impact on the reaction dynamics, and therefore make possible task-specific organic ionic liquids.(40) The design of task-specific RTILs may depend on the dynamics of molecules in distinct environments in addition to the specific substrate interactions. 
The addition of co-solvents to RTILs is an important area of study.(41) To reduce the often high viscosity of most RTILs, low viscosity co-solvents are frequently added.(42,43) One of the most common co-solvents, water, is especially important, in part due to the hygroscopic nature of many RTILs and the significant effect a small amount of water can have on properties such as viscosity and conductivity.(44) In applications, RTILs will have some water in them because of the difficulties and expense of producing dry ionic liquids. Thus, the question of the role of water in RTILs on the liquid's structure and dynamics is of importance. Simulations of the addition of water to various RTILs indicate that adding water promotes the alkyl tail aggregation up to a water mole fraction of $\sim 0.75$ and then proceeds to break it up as the volume fraction of water becomes larger. $(45,46)$ It has been observed that 1-decyl-3-methylimidazolium bromide or nitrate $\left(\mathrm{DmImBr}\right.$ or $\left.\mathrm{DmImNO}_{3}\right)$ RTILs gelled at a similar mole fraction of water(47) and the same behavior has been observed for 1-methyl-3-octylimidazolium chloride (OmImCl).(48) The proposed hexagonal mesophase structure for the gel(47) has been observed in simulations.(49) The structural changes in water/RTIL co-solvent mixtures should significantly alter reaction rates and thus the dynamics of such binary mixtures are important.

Here a variety of experiments will be presented that illuminate many of the properties and features of RTILs. First, the orientational relaxation dynamics of fluorescent probes were studied by measuring their time dependent orientational anisotropy.(36,50) Two probe molecules were employed. One is nonpolar and was located in the organic alkyl regions of the RTILs. As the alkyl chains got longer, the friction coefficients approach those of a long chain hydrocarbon. The other fluorescent 
probe was an anion and was located in the ionic regions. Its orientational relaxation was strongly affected by interactions with the surrounding cations. The results are consistent with different elements of rotational friction available to solutes depending on their chemical nature because of the ionic liquids' nanoscopic heterogeneous structures. Information on the influence of lithium cations on RTIL structure was obtained from time resolved fluorescence anisotropy measurements of a nonpolar chromophore that is located in the alkyl regions of the RTIL. As the concentration of $\mathrm{Li}^{+}$was increased, the rotational friction experienced by the chromophore changes, demonstrating that the addition of lithium cation to the ionic regions changes the structural arrangement of the alkyl chains in the organic regions.

Optical heterodyne detected optical Kerr effect (OHD-OKE) experiments were used to study the bulk orientational relaxation dynamics of several pure dry RTILs over a wide range of temperatures. $(51,52)$ The experiments followed the orientational relaxation from hundreds of femtoseconds to a hundred nanoseconds. The results were analyzed using schematic mode coupling theory. The orientational relaxation is complex, consisting of several power laws at short time and ending with an exponential decay, which is the final complete randomization of the orientations. The power laws reflect the dynamics on time scales during which a molecule is "caged" by the surrounding molecules and is not undergoing orientational diffusion. The functional form of the RTIL dynamics and their temperature dependence are indistinguishable from a wide range of organic liquids. These results show that in spite of the nanoscopic heterogeneity of RTILs, they have dynamical properties that are no different from organic liquids such as dibutylphthalate $(53,54)$ or acetylsalicylic acid.(54) 
In contrast to the pure dry RTILs, the OHD-OKE experiments reveal dramatic difference in dynamics when even small amounts of water are added.(55,56) For chain lengths of hexyl and longer, there is clear evidence that water in the ionic regions produces structuring of alkyl chains that dramatically slows orientational relaxation and changes its fundamental nature. For RTILs that gel, the results indicate that gelation is preceded by water induced changes in structural dynamics.

\section{Time Resolved Fluorescence Probes of the Organic and Ionic Regions of RTILs}

The nanoscopic heterogeneity picture of RTILs implies distinct organic and ionic regions. An important question is how closely do the organic regions, which consist of the alkyl tails of the cation head groups, resemble a hydrocarbon liquid, and how does an ionic solute interact with the ions in the ionic regions. To examine these questions, the orientational relaxation dynamics of perylene and sodium 8-methoxypyrene-1,3,6sulfonate (MPTS) (see Figure 1) were determined using time dependent fluorescence anisotropy measurements.(36) Perylene is neutral and nonpolar. In contrast, MPTS has three negatively charge sulfonate groups. These two probe molecules locate in the distinct regions of the RTIL heterogeneous structure.(36) The series of ionic liquids of alkyl-substituted imidazolium bis(trifluoromethanesulfonyl)imide (abbreviated as $\mathrm{C}_{\mathrm{n}} \mathrm{mIm}^{+} \mathrm{NTf}_{2}^{-}$) was chosen as a class of relatively hydrophobic ionic liquids that can be obtained at high purities. They have fairly low viscosities and demonstrate prototypical ionic liquid behavior.

For each liquid, the orientational relaxation and the viscosity were measured as a function of temperature. These measurements permit the details of the probe interactions with its environment to be explicated. The triply charged fluorophore and the neutral 
non-polar fluorophore are similar in size (see Figure 1) and were studied in a series of RTILs with increasing hydrocarbon chain lengths. The temperature dependent studies of the orientational relaxation enabled the local friction to be extracted from the data. By measuring the orientational relaxation on the series of liquids, it was possible to examine changes in the nature of the local environments experience by the two types of probe molecules. Previous fluorescence studies of orientational relaxation in RTILs have seen Debye-Stokes-Einstein (DSE) behavior(57) and possibly deviations from DSE behavior.(17,58,59) Most of the studies examined very polar and charged dye molecules. Mali, et al. studied a charged dye molecule and a non-polar chromophore in a single RTIL.(58)

The sample preparation has been described in detail.(36) All of the samples were of the high purity and very low water content. The samples were handled in a glove box to avoid picking up water during sample preparation in $1 \mathrm{~cm}$ quartz spectroscopic cuvettes. The viscosities of all of the samples were measured as a function of temperature.(36)

The time dependent fluorescence measurements were made with time correlated single photon counting (TCSPC).(36,50). The samples were housed in a computer controlled cryostat stable to $\pm 0.1 \mathrm{~K}$. The instrument response was measured to be $\sim 40 \mathrm{ps}$. Time dependent fluorescence data were collected at each temperature (298 K to $333 \mathrm{~K}$ in $5 \mathrm{~K}$ increments) in parallel, perpendicular, and magic angle $\left(54.7^{\circ}\right)$ polarizer orientations. Samples were excited at $380 \mathrm{~nm}$ for MPTS and $409 \mathrm{~nm}$ for perylene. The excitation pulses were single pulses selected at $4 \mathrm{MHz}$ rate from a Ti:Sapphire oscillator and doubled to the excitation wavelengths. Fluorescence, after passing through an 
appropriate long pass filter to remove excitation light, was observed at $420 \mathrm{~nm}$ for MPTS and $469 \mathrm{~nm}$ for perylene with a channel plate detector on a monochromator.

The fluorescence experiments measure the time dependent anisotropy, $r(t)$, defined as

$$
r(t)=\frac{I_{\|}(t)-I_{\perp}(t)}{I_{\|}(t)+2 I_{\perp}(t)},
$$

with $I_{\|}$and $I_{\perp}$ the time dependent fluorescence intensities polarized parallel and perpendicular to the excitation beam, respectively. The functional form of the fluorescence anisotropy decay for the most general case of orientational diffusion has been presented.(60) In the experiments described here, the observed exponential decays (bi-exponential for perylene and single exponential for MPTS, see below) were related to hydrodynamic properties through the Debye-Stokes-Einstein relation:

$$
\tau=\frac{1}{6 D}=\frac{\eta V f C}{k T}
$$

where $\tau$ is the measured orientational relaxation time constant, $D$ is the orientational diffusion constant, $\eta$ the viscosity, $k$ is the Boltzmann constant, $V$ is the molecular volume, $C$ is a friction coefficient, and $f$ is the shape factor. For a given molecule, the friction coefficient will contain information on the rotational friction experienced by the molecule. For large particles, the stick boundary condition $(C=1)$ almost always holds. For rotators of similar size to the solvent molecules, the continuum approximation of the solvent begins to break down. This is manifest as a decrease in friction toward the slip limit, for which the value of $C$ depends on the geometry of the rotator.

Figure 1 shows the structures and dimensions of the fluorophores used in this study. For both molecules, the shape is close to ellipsoidal based on a geometrically 
minimized structure. Using the van der Waal radii of the constituent atoms, molecular volumes of $225 \AA^{3}$ for perylene and $343 \AA^{3}$ MPTS were calculated. From the molecular dimensions, the shape factors(61) and theoretical slip friction coefficients $(62,63)$ can also be generated. These quantities have been reported for perylene and MPTS.(36)

\section{A. Perylene}

Due to the high $\mathrm{D}_{2 \mathrm{~h}}$ symmetry of perylene, the equation for the fluorescence anisotropy simplifies to a bi-exponential. The orientational motions of perylene are very different in the plane of the molecule and out of the plane. The out of plane motions requires a great deal of solvent to be "pushed" out of the way, in contrast to the in plane motion. In plane rotation occurs about the axis normal to the plane of the molecule, the $\mathrm{z}$ axis in Figure 1. Perylene is modeled as an oblate spheroid rotator, that is the diffusion constants $D_{x}=D_{y}<<D_{z}$, using the coordinate system of Figure 1. Then the anisotropy decay is(36)

$$
r(t)=0.1 e^{-\left(2 D_{x}+4 D_{z}\right) t}+0.3 e^{-6 D_{x} t} .
$$

Anisotropy studies of perylene have often encountered a well-known "anisotropy deficit," that is, an initial anisotropy of less than the theoretical value of 0.4.(64) Then the prefactors in Equation 3 have smaller than the theoretical values of 0.1 and 0.3 , which does not impact the information content of the experiments.

To extract the rotational diffusion constants, measured time dependent anisotropies were fit to a bi-exponential function convolved with the instrument response using nonlinear least squares. From the fits, the appropriate diffusion constants can be obtained from linear combinations of the exponential time constants using Equation 3. Figure 2 shows the temperature dependent orientational relaxation decay constant, 
$\tau=1 / 6 D$, where here $D$ is the average diffusion constant. The lines through the data in the figure are fits that confirm Debye-Stokes-Einstein behavior.

The results of the analysis are presented in Table 1 as a list of the experimentally determined average friction coefficients ( $C$ in Equation 2). As the alkyl chain length increases, the friction coefficient approaches that of perylene in paraffin oil, which is the first indication that the alkyl tail regions of the RTIL behaves like a hydrocarbon when the tails are sufficiently long.

From the values listed in Table 1, perylene is found to rotate with slip to sub-slip boundary conditions. Rotational dynamics in the sub-slip regime have been studied previously $(65,66)$ and are often attributed to complex solvation structure in the vicinity of the probe molecule. This structuring can lead to effective void spaces through which the molecule can rotate with little hindrance, leading to a decrease in rotational friction below slip. This picture was used to describe the rotational dynamics of tetracene in alcohols.(67)

In the perylene/RTIL experiments, the degree of sub-slip increases with increasing alkyl tail length, approaching the value for perylene in paraffin oil at $\mathrm{C}_{8} \mathrm{mIm}^{+}$. Due to the high symmetry of perylene, the bi-exponential experimental decays permit the rotational diffusion to be further decomposed into components of rotation in-plane $\left(D_{z}\right)$ and out-of-plane $\left(D_{x}\right)$. The results are presented in Table 2 as the ratio of the appropriate friction coefficient to its theoretical value for slip boundary conditions. A value of 1 means that the measured and calculated values are the same. A ratio larger than 1 indicates that the measured value is slower than slip, and a ratio smaller than 1 shows that the experimental value is faster than slip. The trends displayed in Table 2 demonstrate 
that as the length of the alkyl chain length increases, relative to the theoretical slip values, the in plane rotational diffusion becomes faster with a concurrent slower rotational diffusion out of plane. Both the in-plane and out of plane rotational diffusion are converging on the values found in the paraffin oil. The increase of in-plane rotational diffusion constant with alkyl chain length is consistent with previous work on perylene in n-alkanes. For longer alkanes, the results are interpreted as a partial alignment of the alkane chains along the long axis of the perylene $(68,69)$. This interpretation is supported by studies of gas phase van der Waal complexes(70). Therefore, the orientational relaxation trends with RTIL chain length demonstrate that the environment experienced by perylene is increasingly like that of a partially ordered alkane. The trend in Table 2 suggests by a chain length of ten carbons, the alkyl environment would be virtually indistinguishable from a long chain alkane.

These convergent trends of perylene demonstrate that the solvation environment of perylene in the ionic liquids becomes more alkane-like as the alkyl chain increases in length. In the context of ionic liquid structure, one might concluded that the aliphatic moiety is dominant in solvating the perylene. This is consistent with simulations on solvation in ionic liquids(71,72), as well as optical Kerr effect experiments.(73) The selective solvation constitutes an effective partitioning of the perylene into the hydrophobic regions of the bulk ionic liquid structure. The sub-slip rotational behavior is describing the structure and microviscosity of the hydrophobic pockets. It is important to note the apparent lack of a "turn-on" of nanoscale segregation. Both the in plane and out of plane friction coefficients change continuously toward those of perylene in paraffin oil. 
This interpretation leads to several interesting possibilities. Although not necessarily of the same origin, void spaces in RTILs have been evoked when discussing the solubility of $\mathrm{CO}_{2}$.(74) The directionality of the rotational friction (see Table 2) may provide insight into anomalous Diels-Alder reactions in RTILs.(38) Tiwari and coworkers find that the rate of an intra-molecular Diels-Alder reaction involving a nonpolar reactant does not change significantly in going from $\mathrm{C}_{4} \mathrm{mIm}^{+} \mathrm{NTf}_{2}^{-}$to $\mathrm{C}_{4} \mathrm{mIm}^{+}$ $\left[\mathrm{BF}_{4}^{-}\right]$, even though the viscosity changes substantially. The reaction involves the rotation of the dienophile over the plane of the molecule. This is the direction of rotation in $\mathrm{C}_{4} \mathrm{mIm}^{+}$that is most strongly sub-slip, indicating a relatively unhindered rotation produced by the ionic liquid structure that should be relatively insensitive to the anion.

\section{B. MPTS}

The anisotropy decays of MPTS in all liquids studied are single exponential decays, reflecting the lower symmetry of MPTS compared to perylene and indicating similar MPTS rotational diffusion coefficients for the various axes. The decay times vs. $\eta / k T$ are shown in Figure 3 for three RTILs (A), DMSO, and water (B). MPTS is essentially insoluble in $\mathrm{C}_{8} \mathrm{mIm}^{+}$, so this liquid could not be studied. Because of the low viscosity, the data for MPTS in DMSO and water have very fast decays times. The data for these two liquids are shown in Figure 3B. Note the differences in the axes between Figures 3A and B. As is the case for perylene, the MPTS temperature dependent data in each liquid is linear within a very small error, demonstrating Debye-Stokes-Einstein behavior.

The friction coefficients for the five liquids are given in Table 3. The friction coefficient for stick boundary conditions is 1 . In contrast to the perylene (see Table 1), 
the MPTS friction coefficients are all greater than 1 for the ionic liquid samples (Table 3), indicating super-stick boundary conditions. MPTS in water and DMSO were used as control solvents for comparison. MPTS in these two solvents yield close to stick boundary conditions. Super-stick conditions observed in the RTILs are traditionally explained using either the "solventberg" model(75) or Nee-Zwanzig dielectric friction(76) The former assumes specific solvent-solute interactions anchor solvent molecules of non-negligible size to the solute, effectively increasing the rotator volume. The latter involves the electrostatic torque between a dipole and the reactive field of the surrounding dielectric cavity. For alcohol solvents that can hydrogen bond to the solute, research on the relative contributions of the two mechanisms has indicated that the "solventberg" effect dominates.(77) Detailed calculations of the dielectric friction model show that it cannot explain the MPTS in RTIL results given in Table 3.(36)

The 3 formal -1 charged sulfonate groups should interact very strongly with the charged cations of the ionic liquid. The MPTS orientational relaxation data show highly hindered motion that is likely caused by strongly associated solvent molecules. The most obvious possibility is that RTIL cations are bound to the sulfonate anions. A simple calculation can estimate the number of associated solvent molecules that would be necessary to obtain the results for the RTILs given in Table 3. Using the cation van der Waals volumes, the results yield 3.0, 2.8, and 3.1 cations are "attached" to each MPTS in $\mathrm{C}_{2} \mathrm{mIm}^{+}, \mathrm{C}_{4} \mathrm{mIm}^{+}$, and $\mathrm{C}_{6} \mathrm{mIm}^{+}$, respectively. These numbers, which are all basically 3 , correspond to the number of sulfonate groups on MPTS, supporting the idea that cation coordination is responsible for the slow (super-stick) orientational relaxation of MPTS in the RTILs. Furthermore, the friction coefficient increases as the RTIL cation alkyl chain 
increases. The increase in friction is consistent with larger and larger cations being strongly bound to the MPTS sulfonate groups as RTIL solvent goes from $\mathrm{C}_{2} \mathrm{mIm}^{+}$to $\mathrm{C}_{4} \mathrm{mIm}^{+}$to $\mathrm{C}_{6} \mathrm{mIm}^{+}$.

The results presented above are consistent with a picture of RTILs in which there is nanoheterogeneity consisting of ionic regions and alkyl regions. Based on measurements of rotational friction for perylene and MPTS, two distinct rotational environments were found. Perylene undergoes orientational relaxation in the slip to subslip regime, that is orientational relaxation is faster than predicted by hydrodynamic theory. As the alkyl chain length of the cation increases, the in plane and out of plane orientational relaxation friction coefficients converge to those of perylene in paraffin oil (see Table 2). Perylene partitions into alkane-like environments.(35) The trends in the in plane and out of plane friction coefficients indicate a degree of alkyl chain alignment along the perylene long molecular axis.

MPTS undergoes orientational relaxation with super-stick boundary conditions, in contrast to MPTS in DMSO and water, which show approximately stick boundary conditions. The slow orientational relaxation of MPTS in the RTILs cannot be ascribed to a non-specific strong solute solvent interaction.(36) The results indicate that three RTIL cations are bound to the three MPTS sulfonate anions, and the entire assembly undergoes orientational diffusion. The increased volume of MPTS accounts for the slow orientational diffusion. The friction increases with increasing size of the solvent cation, which is consistent with the picture of cations bound to the MPTS sulfonate anion.

\section{Addition of Lithium Cations}


Relative to the neat liquids, the viscosities of solutions of lithium salts in RTILs increase significantly.(50,78,79) The results of experimental studies(80-83) and molecular dynamics simulations $(81,82,84)$ suggest that RTIL anions aggregate around the $\mathrm{Li}^{+}$ions, producing the increase in viscosity. Because of the importance of lithium ion transport to battery operation, the influence of lithium ions on RTIL structure and dynamics is a topic of considerable interest. The alkylimidazolium bistriflimide (bis(trifluoromethyl)sulfonyl imide) ionic liquids are attractive for battery applications because of their relatively low viscosities, as well as good electrochemical stability(85) and broad operating temperature ranges.

The influence of lithium cations on the structure of the alkyl chains was investigate by using perylene as a dynamical probe of the alkyl region structure of 1butyl-3-methylimidazolium bis(trifluoromethyl)sulfonyl imide ([BmIm] $\left.\left[\mathrm{Tf}_{2} \mathrm{~N}\right]\right)$ as a function of the $\mathrm{Li}^{+}$concentration.(50) The methodology is the same as used in the studies described above that examined the influence of chain length on the structure of the alkyl regions by measuring the temperature dependent orientational relaxation of perylene, which gave the friction coefficients.

To show the nature of the trends with $\mathrm{Li}^{+}$concentration, the three diffusion constants, in-plane, out-of-plane (see Figure 1A), and the average diffusion constants are displayed for a single temperature, $298 \mathrm{~K}$, in Figure 4A. The dashed lines are aids to the eye. With the exception of the points at $\chi=0.1$, the points all fall very close to the lines. The points for $\chi=0.1$ are probably off the lines because of experimental error, but the possibility that the diffusion constants do not change in a linear manner with $\mathrm{Li}^{+}$mole fraction cannot ruled out. As is clear from the figure, the orientational diffusion becomes 
slower with increasing lithium ion concentration. Orientational diffusion constants for solute molecules generally depend inversely on solution viscosity, and the viscosity of $[\mathrm{BmIm}]\left[\mathrm{Tf} f_{2} \mathrm{~N}\right]$ solutions increase with $\mathrm{LiTf}_{2} \mathrm{~N}$ concentration, as shown in Figure 4B. The temperature-dependent viscosities are fit very well with single exponentials with a small offset ( $\mathrm{R}^{2}$ greater than 0.999 for each $\mathrm{LiTf}_{2} \mathrm{~N}$ concentration). The parameters for the exponential fits have been given previously,(50) so that the viscosity over the range of temperatures studied can be obtained for the various $\mathrm{Li}^{+}$concentrations.

In Figure 5, the experimental values of the orientational relaxation times, $\tau$, taken over a range of temperatures for perylene for each lithium/RTIL solution are plotted against $\eta / k T$. To make it easier to see the nature of the results and the trends, the figure shows the data rescaled and shifted. The true slopes of the data, which represent the proportional dependence of $\tau$ on $\eta / k T$, have been preserved in all cases. Linear leastsquares fits gave $\mathrm{R}^{2}$ of greater than 0.98 for each sample. The linearity of the plots confirms hydrodynamic behavior. To obtain actual values of $\tau$ for the corresponding $\eta / k T$ from Figure 5, find the point of interest on the plot and the associated shift and scale values from the inset. Subtract the shift value from $\tau$ and then divide both $\tau$ and $\eta / k T$ by the scale factor.

As can be seen in Figures 4A and 5, as the mole fraction of $\mathrm{Li}^{+}$increases the diffusion constants become slower (the orientational relaxation time constants become longer). This is expected as increasing $\chi\left(\operatorname{LiTf}_{2} \mathrm{~N}\right)$ increases the viscosity at any given temperature. However, to determine whether the slower orientational diffusion of perylene with increasing lithium ion concentration is solely a consequence of increasing viscosity or indicates a structural change in perylene's solvation environment, it is 
necessary to analyze the trends in the diffusion constants with temperature and viscosity. A useful approach is to consider the unitless friction coefficient $C$, which represents the coupling between the solute and the solvent and is independent of temperature and viscosity for rotators that display hydrodynamic behavior. $(62,86)$ This is the same method used for the analysis in Section II.A. Figure 5 shows that the orientational relaxation of perylene in all of the samples is hydrodynamic. The friction coefficient $C_{i}$ for rotation about the $i^{\text {th }}$ axis of an oblate ellipsoid is related to the associated $D_{i}$ as $(62)$

$$
C_{i}=\frac{k T}{\eta V D_{i}}
$$

where $V$ is the volume of the solute. $C_{i}$ can be calculated from the measured temperaturedependent values of $D_{i}$ and $\eta$. For perylene in each solution, the ratios of the experimental in-plane and out-of-plane friction coefficients to their theoretical slip values, were calculated from the dimensions of the rotator.(62,63) Figure 6 is a plot of the friction coefficients with a linear fit to the data points. The friction coefficients change significantly with lithium ion concentration. The values of the friction coefficients are given elsewhere.(50) The fact that the coupling between solute and solvent, as quantified by the friction coefficients, is not constant with $\operatorname{LiTf}_{2} \mathrm{~N}$ concentration demonstrates that adding lithium ions has an effect on the alkyl region of the RTIL beyond altering the solutions' bulk viscosities. As shown in Figure 6, both in-plane and out-of-plane orientational friction coefficients decrease as the $\mathrm{Li}^{+}$concentration increases. The inplane friction coefficient $\left(C_{z}\right)$ is greater than the theoretical slip value with no lithium cations in the solution. As $\operatorname{LiTf}_{2} \mathrm{~N}$ is added, the in-plane friction coefficient falls, becoming subslip for concentrations above $\chi\left(\operatorname{LiTf}_{2} \mathrm{~N}\right)=0.2$. The out-of-plane friction coefficient $\left(C_{y}\right)$ is subslip even for $\chi=0$. It becomes increasingly subslip as $\chi\left(\operatorname{LiTf}_{2} \mathrm{~N}\right)$ 
increases. $C_{z}$ decreases more in relative terms than does $C_{y} ; C_{z}$ decreases by a factor of $\sim 6$ from $\chi\left(\operatorname{LiTf}_{2} \mathrm{~N}\right)=0$ to 0.4 , while $C_{y}$ decreases by a factor of $\sim 3$. At high lithium ion concentration, $\chi\left(\operatorname{LiTf}_{2} \mathrm{~N}\right)=0.4, C_{z}$ is approaching $C_{y}$.

The decreases in the friction coefficients, $C_{z}$ and $C_{y}$, show that changes in the liquid structure in the RTIL alkyl region in response to increased lithium concentration result in less hindered orientational diffusion both in and out of the plane of perylene after adjusting for the increased viscosity of the RTIL. This is in contrast to the effect of increasing alkyl chain length on perylene in 1-alkyl-3-methylimidazolium bistriflimides discussed in Section II.A.(36) As the length of the cation's alkyl tail increased, the dynamics of the perylene probe converged toward those of an ordered alkane, with the inplane $C_{z}$ (rotation about the $z$ axis) decreasing and becoming subslip while the out-of plane $C_{y}$ (rotation about the $y$ axis) increased. Therefore, the structural changes in the alkyl region in response to lithium ions are qualitatively different from those that result from changing the alkyl chain length.

The trends in the orientational dynamics of perylene can provide insights into the changing nature of the liquid structure of the RTIL alkyl regions as lithium cation is added. Slip boundary conditions for orientational diffusion of solute molecules apply when the interactions with the solvent are not strong, e.g., no solute-solvent hydrogen bonding. Slip boundary conditions are frequently seen for solvents that are similar in size or larger than the solute molecules.(87) Under these conditions, the rotating solute does not "drag" solvent with it. A hypothetical spherical solute with slip boundary conditions would rotate freely. However, for non-spherical solutes with slip boundary conditions, the solute must still displace solvent molecules that are within its swept volume as it 
rotates, which gives rise to the friction. In some cases, $C$ may be less than the theoretical slip value; such subslip behavior has generally been explained by the presence of void spaces or excess free volume in the local liquid structure around the rotator, giving lower than average solvent density within the swept volume of the rotating solute. $(65,67,74)$

Figure 6 shows that for the pure liquid $\left(\right.$ no $\mathrm{Li}^{+}$), the in-plane friction is greater than the out-of-plane friction. As discussed above, the values in Figure 6 are the ratios of the measured friction coefficients to the theoretical values for perylene modeled as an oblate ellipsoid. A value of 1 means the measured friction coefficient is the same as the value for the perylene model under slip conditions. The slip condition can be thought of as occurring in a hypothetical ideal solvent that does not have a particular local structure that is different for in-plane and out-of-plane. The only source of friction is sweeping out the volume of solvent molecules by the rotator. The model oblate ellipsoid in this ideal solvent would have friction coefficient ratios in-plane and out-of-plane that are both 1. The fact that the in-plane ratio is greater than slip and substantially larger than the out-ofplane ratio, which is subslip, indicates that perylene finds itself in a structured environment even in the absence of lithium cation. The structure is such that relative to the theoretical slip calculation, it is more difficult for perylene to push the alkyl tails of the RTIL out of the way when rotating in the plane of the molecule than out of the plane. This is in contrast to perylene embedded in the alkyl chains of paraffin oil discussed in Section II. A for which the two friction coefficients ratios are almost identical and both are subslip.(36)

Figure 6 show that increasing the $\mathrm{Li}^{+}$concentration causes both friction coefficients to decrease. These decreases as $\chi\left(\operatorname{LiTf}_{2} \mathrm{~N}\right)$ increases clearly demonstrate that 
putting $\mathrm{Li}^{+}$in the ionic regions of the $\mathrm{RTIL}$ changes the structure of the alkyl regions. It is becoming easier for perylene to undergo rotational diffusion (after accounting for the increased viscosity of the solutions) as the $\mathrm{Li}^{+}$concentration increases. The change is more pronounced for the in-plane motion than the out-of-plane motion, and both motions become substantially subslip at $\chi\left(\operatorname{LiTf}_{2} \mathrm{~N}\right)=0.4$.

Adding $\mathrm{Li}^{+}$to the ionic region can change its organization causing the configuration of the BmIm cations to alter as the $\mathrm{Li}^{+}$concentration grows. Changing the configuration of imidazolium cations in the ionic regions alters the organization of the butyl chains that solvate perylene in the alkyl regions. The large decrease in the friction coefficients implies that the chain packing density around the perylene is reduced, but in an anisotropic manner. The structuring in the ionic regions may generate a good deal of free volume in the alkyl regions, resulting in reduced hindrance of perylene orientational diffusion.

\section{Bulk Dynamics of RTILs and the Influence of Water - OHD-OKE Experiments}

\section{A. Pure RTILs}

The last section clearly demonstrated the nanoscopic heterogeneity of the RTILs and explicated aspects of interactions with solutes that selectively locate in the alkyl and ionic regions. A remarkable aspect of pure RTILs is that they have bulk dynamical properties that in many respects are indistinguishable from those of relatively simple organic liquids. The dynamics of RTILs were investigated using optical heterodyne detected optical Kerr effect experiments.(88-90) The results are analyzed with schematic mode coupling theory. $(51,52)$ 
The temperature dependent orientational relaxation of RTILs and normal liquids has a complex time dependence that spans a few hundred femtoseconds to hundreds of nanoseconds and can exhibit five decades of signal decay. As discussed below, the dynamics involve several power laws and, at long time, a final exponential decay. The power laws reflect the dynamics on time scales during which a molecule is "caged" by the surrounding molecules and is not undergoing orientational diffusion. The final exponential decay is the orientational diffusion complete randomization of the liquid. To observe the complete time dependence requires a method that can span the necessary range of times and signal amplitudes. The OHD-OKE experiment has the necessary time and amplitude dynamic ranges to observe the full range of orientational relaxation.

The OHD-OKE experiment measures the time derivative of the polarizabilitypolarizability correlation function, which is directly related to the data obtained from depolarized light scattering experiments by Fourier transform.(91-94) However, the OHD-OKE experiments have the advantage of working in the time domain, which provides a wider dynamic range in both signal amplitude and time span. The polarizability-polarizability correlation function is essentially the second Legendre polynomial orientational correlation function except at short time (less than a few ps) where "collision induced" or "interaction" effects (density fluctuations) can also contribute to the decay.(92-95) In the experiments, a pump pulse creates an optical anisotropy and a probe pulse is used to heterodyne detect its decay at variable time delays. To observe the full range of liquid dynamics, at each temperature several sets of experiments were performed with different pulse lengths and delays. For times $\mathrm{t}<30 \mathrm{~ns}$, a $5 \mathrm{KHz}$ mode-locked Ti:Sapphire laser/regenerative amplifier system was used $(\lambda=800$ 
$\mathrm{nm}$ ) for both pump and probe. The pulse length was varied by from 75 fs to $100 \mathrm{ps}$ as the time scale of the measurement increased to improve the signal-to-noise ratio. For longer times, a CW laser was used for the probe, and a fast digitizer ( 1 ns per point) recorded the data. The scans taken over various time ranges overlapped substantially, permitting the data sets to be merged by adjusting only their relative amplitudes. The details of the experimental setup can be found in previously published work. $(96,97)$ The latest version of the experiments uses a four pulse phase cycling system, in which the phase of the probe and the polarization of the pump are cycled every shot at $5 \mathrm{kHz}$ using Pockels cells.(56) This method greatly improves the signal-to-noise ratio beyond that achieve in the initial experiments described here. The new method is used to obtain some of the data presented below.

Figure 7 shows the nature of the data. The date in Figure7A is for dibutylphthalate, a normal organic liquid.(54) The data in Figure 7B is for the RTIL butylmethylimidazolium tetrafluoroborate $\left(\mathrm{BmImBF}_{4}\right)$. A number of normal liquids $(98,99)$ and $\operatorname{RTILs}(51,52,83)$ have been studied using OHD-OKE experiments, and for the pure liquids, they all have the identical functional form. At short times, the data consist of two power laws, called the intermediate power law and the von Schweidler power law, $(51,98,99)$ which are followed by an exponential decay.(54) There is a third power law predicted by MCT, called the fast $\beta$ process $(89,100)$ that is not shown in Figure 7. In the supercooled liquid literature, the final exponential decay is referred to as $\alpha$ relaxation. The dashed red line through the $\mathrm{BmImBF}_{4}$ data is a fit to the empirical fitting function, which is based on MCT analysis, 
The second and third power laws are the intermediate power law and the von Schweidler power law, respectively. This function, which captures the form of the numerical solutions to the schematic mode coupling description (see below) is useful as it permits the power law exponents and the exponential decay time constant to be extracted. In fitting data, it is necessary to do a global fit to Equation 5 to obtain the exponential time constant as the power laws influence the decay even in the exponential region of the data. An important point shown in Figure 7 is that the nanostructuring of the RTIL does not change the functional form of the overall bulk liquid orientational relaxation dynamics.

Temperature dependent experiments with analysis using mode coupling theory have been performed on a number of RTILs. $(51,52,83)$ Figure 8 shows data for Npropyl-3-methylpyridinium bis(trifluoromethylsulfonyl)imide (PMPIm) at $T=233 \mathrm{~K}$, where the liquid is deeply supercooled.(52) For supercooled van der Waals liquids, the exponents $z$ and $b$ are found to be temperature independent.(98,99) The exponential $\alpha$ relaxation, however, is highly temperature dependent, with the time constant $\tau_{\alpha}$ increasing dramatically as the glass transition is approached from above.(98) The lack of temperature dependences for the $\mathrm{z}$ and $\mathrm{b}$ exponents is also found for RTILs, as is the strong temperature dependence of the $\alpha$ relaxation. $(51,52)$ The inset shows the long time portion of the decay on a semi-log plot. The long time portion of the data is linear, showing that it is an exponential decay. Figure 9 displays the PMPIm OHD-OKE data and fits using Equation 5 for the range of temperatures studied. From the top to the bottom, the curves are arranged in the order of increasing temperatures. The curves have been off-set along the vertical axis for clarity of presentation. The lowest temperature 
data span the longest time window because the final complete $\alpha$ structural relaxation slows down as the temperature is decreased. The functional form of OHD-OKE decay curves does not change when the temperature is varied, but the numerical values of some of the fitting parameters change with temperature.

The temperature dependent data permits a detailed comparison to the predictions of schematic mode coupling theory. The numerical solutions to the MCT coupled differential equations that describe the density and orientational correlations functions have been shown to very accurately reproduce the complete time dependence of the RTIL OHD-OKD data over the full ranges of temperatures studied.(51,52,83) MCT also predicts certain scaling relations(101) for the supercooled liquids as they approach the MCT critical temperature $T_{c} . T_{c}$ is called the "ideal" glass transition temperature because in schematic MCT, the $\alpha$-relaxation is predicted to diverge at $T_{c}$, becoming infinitely slow. The cessation of complete structural relaxation predicted by MCT to occur at $T_{c}$, that is, the liquid becomes non-ergodic, was thought to signal the glass transition. However, experimentally the $\alpha$-relaxation time does not become infinitely slow at $T_{c}$ and continues to increase below $T_{c}$. The experimental glass transition temperature $T_{g}$ is usually $15 \%$ to $20 \%$ below $T_{c}$. Previously reported OHD-OKE data on several supercooled organic molecular liquids obey the MCT scaling relations at temperatures above $T_{\mathrm{c} .}(98)$

As a test of MCT in RTIL systems, the standard MCT scaling law analysis was performed on PMPIm and other supercooled RTILs. The MCT scaling relation for the $\alpha$ relaxation time constant, $\tau_{\alpha}$, gives scaling with temperature as(101)

$$
\tau_{\alpha} \propto\left(T-T_{C}\right)^{-\gamma} \text { or } \tau_{\alpha}^{-1 / \gamma} \propto T-T_{C},
$$


with

$$
\gamma=(a+b) / 2 a b,
$$

and $a$ and $b$ are MCT parameters, which are related to each other via

$$
\Gamma^{2}(1-a) / \Gamma(1-2 a)=\Gamma^{2}(1+b) / \Gamma(1+2 b),
$$

where $\Gamma$ is the gamma function and $b$ is the von Schweidler power law exponent, which is obtained experimentally. The von Schweidler power law amplitude $d$ (Equation 5) scales with temperature as,(101)

$$
d \propto\left(T-T_{C}\right)^{\delta} \text { or } d^{1 / \delta} \propto T-T_{c},
$$

with

$$
\delta=(a+b) / 2 a
$$

Using the value 0.49 for $b$ determined from the fits in Figure 9, $a$ is found to be 0.28 from Equation 5. Then $\gamma$ and $\delta$ are 2.79 and 1.36 respectively using Equations 7 and 10 .

Figure 10A displays the rectification diagram for $\tau_{\alpha} .(52)$ The experimental data points (the open circles) fall on a line indicating the validity of MCT scaling relationship over a significant temperature range. The solid line is a linear fit to Equation 6 using $T_{c}$ and a proportionality factor as adjustable parameters. The extrapolation of the calculated line to its intersection with the abscissa yields $T_{c}=231 \mathrm{~K}$.

The temperature dependence of the von Schweidler power law amplitude $d$ (plotted as $d^{1 / \delta}$ ) is shown in figure 10B. A linear fit using Equation 9 is shown as the solid line. $T_{c}$ is found to be $231 \mathrm{~K}$, consistent with the result determined from $\tau_{\alpha}$. The ratio $T_{g} / T_{c}=0.81$. For comparison, the ratios for 2-biphenyl phenol, ortho-terphenyl, salol, dibutylphthalate, benzophenone, and acetylsalicylic acid are $0.82,0.83,0.85,0.79$, 
0.85 , and 0.87 , respectively. Therefore, the ratio of the RTIL falls within the range of the ratios for common molecular liquids.

The results shown above for PMPIm and for several other RTILs bring out an important aspect of RTILs. The schematic MCT, which has been very successful in describing van der Waals supercooled liquids, works exceeding well for RTILs. The functional form of the decays curves and the scaling relations are the same for van der Waals liquid and RTILs. Schematic MCT is a theory of homogeneous liquids. There is nothing in it that would take into account the nanoscopic heterogeneity of RTILs. Therefore, very detailed consideration of the temperature dependence of the dynamics of pure bulk RTILs show no differences from homogeneous organic liquids.

\section{B. The Influence of Water and Chain Length on RTIL Dynamics}

As discussed briefly in the Introduction, co-solvents(41-43) and particularly water(44) are important in many applications and properties of RTILs. Voth and coworkers simulated the addition of water to various RTILs and proposed that adding water promotes the alkyl tail aggregation up to a water mole fraction of $\sim 0.75$ and then proceeds to break it up as the volume fraction of water becomes larger. $(45,46)$ Firestone observed that 1-decyl-3-methylimidazolium bromide or nitrate ( $\mathrm{DmImBr}$ or $\mathrm{DmImNO}_{3}$ ) RTILs gelled at a similar mole fraction of water(47) and Bowers noticed the same behavior for 1-methyl-3-octylimidazolium chloride (OmImCl).(48) Later, Bhargava and Klein successfully simulated the hexagonal mesophase structure originally proposed by Firestone.(49) The structural changes in RTIL/water mixtures may significantly influence processes that occur in them. Therefore, the dynamics of such binary mixtures are important. 
In this section, the influence of the addition of water on the dynamics and structure of RTILs is addressed. OHD-OKE experiments as discussed above are employed, but the experimental system uses the new phase cycling method mentioned previously.(56) The experiments were conducted on a series of 1-alkyl-3methylimidazolium tetrafluoroborate RTILs and their mixtures with water. Using the phase cycling method, the decays can be followed from a few hundred femtoseconds up to $\sim 0.4$ microseconds (six decades of time) and over six decades of signal amplitude. The pure RTIL decays are analyzed as two power laws followed by a final exponential decay like those discussed in Section III.A. As water is added, the viscosities of all of the RTIL solutions decrease monotonically. For the shorter alkyl chain samples, and for the lower water concentrations of the longer chain samples, as water is added the final single exponential decay, reflecting the complete randomization of the liquid, becomes faster. However, when water was added to the RTILs with an alkyl tail of hexyl or longer, the long time scale orientational dynamics became bi-exponential. The slowest component of the dynamics for the RTILs with octyl or decyl tails does not obey the Debye-StokesEinstein (DSE) equation, which was attributed to significant local structuring of the alkyl tails. The fast component of the bi-exponential dynamics is assigned to the restricted reorientation of the imidazolium head group (wobbling-in-a-cone). Detailed analysis yielded estimates of the cone angle sampled by the head group prior to complete randomization. The bi-exponential dynamics and non-DSE behavior of the systems indicate that the use of water as an RTIL co-solvent can have effects on reaction rates and other processes that cannot be solely explained by changes in viscosity. 
RTIL/water mixtures were prepared by mass. $\mathrm{HmImBF}_{4}, \mathrm{OmImBF}_{4}$, and $\mathrm{DmImBF}_{4}$ are not fully miscible with water (unlike $\mathrm{BmImBF}_{4}$ ), saturating at water mole fraction of $\sim 0.75$ at $24.5^{\circ} \mathrm{C}$. Water mole fraction is defined as $\mathrm{mol} \mathrm{H}_{2} \mathrm{O} /(\mathrm{mol}$ cation + mol anion $+\mathrm{mol} \mathrm{H}_{2} \mathrm{O}$ ). Water contents were measured with Karl Fisher titration after data acquisition for all samples. For the pure RTILs, the water concentration was 100 ppm or less. Dynamic viscosities for $\mathrm{BmImBF}_{4}$ and $\mathrm{HmImBF}_{4}$ solutions were taken from the literature.(102-104) For the $\mathrm{OmImBF}_{4}$ and $\mathrm{DmImBF}_{4}$ solutions, dynamic viscosities were measured with a Brookfield LVDV-11+P cone and plate viscometer.(55) Multiple literature values were averaged for the pure viscosity of $\mathrm{OmImBF}_{4}(105-110)$ while the pure $\mathrm{DmImBF}_{4}$ viscosity was measured in the nitrogen glove box using the Brookfield viscometer.(55) All measurements were done at $24.5^{\circ} \mathrm{C}$. Imidazolium tetrafluoroborate RTILs are known to hydrolyze slowly in the presence of water.(111) To ensure that the measured dynamics of the binary mixtures were not affected by the hydrolysis products, the OHD-OKE decays of fresh and weeks-old samples were compared. No measurable differences were seen.

Here the primary interest is in the long time scale relaxation that follows the power law decays. The data were fit with Equation 5 using only the second and third power laws and the exponential when the final decay is single exponential. Figure $11 \mathrm{~A}$ shows three representative OHD-OKE decays for three $\mathrm{BmImBF}_{4}$ samples on a logarithmic plot (black solid curves) and fits to the data using Equation 5 ( red dashed curves). The curves have been offset along the vertical axis for clarity. All the $\mathrm{BmImBF}_{4}$ solution OHD-OKE decays, including those not shown, display similar trends, that is, a power law at short time, a power law at intermediate time, and an exponential 
decay at long time. Figure 11B shows three HmImBF OHD-OKE decays (black curves) and fits (red curves). At the lower water concentrations, the final decay is exponential. However at the highest concentration, the final decay is not quite exponential (see arrow). The non-exponential nature of the decay is taken into account in the fits as discussed below. Figure 11C shows three decays for $\mathrm{OmImBF}_{4}$ (black curves) and fits (red curves). At the highest water content shown, the final decay has an inflections point (arrow) and is clearly not exponential. Finally, Figure 12 shows three decays for $\mathrm{DmImBF}_{4}$ (black curves) and the fits (red curves). For this sample, there is a clear break in the data where the decay slows and then decays more rapidly. The inset shows the long time portion of the data with fit for the saturated sample. Note the different time axis for the inset. The decay time constants were obtained as described below.

For the higher water content in longer alkyl tail samples, the OHD-OKE data could not be satisfactorily fit with Equation 5. As can be seen in Figures 11C and 12, the water-saturated samples appear to have a bi-exponential form (see arrows). The biexponential long time behavior is particularly clear in Figure 12. Therefore, these samples were fit with a modified, bi-exponential form,(55)

$$
F(t)=\left[a t^{-s}+d t^{b-1}\right]\left[A_{1} \exp \left(-t / \tau_{1}\right)+A_{2} \exp \left(-t / \tau_{2}\right)\right] .
$$

The fits reproduce the decays well over their entire range. All the exponential amplitudes and decay times from the fits have been reported previously.(55) It is notable that such clearly separated bi-exponential decays (see Figure 12) have never been seen before in optical Kerr experiments. Prior experiments on simple molecular liquids, RTILs (including those with dissolved salts), supercooled liquids, liquid crystals in their 
isotropic phase, and other molecules in aqueous solutions have always shown a long time scale single exponential decay preceded by the power laws. $(51,83,112,113)$

Bi-exponential decays of orientational correlation function are generally recognized as arising from one of two physical situations. In the first case, there are two separate subensembles in the sample that reorient at different rates. The fast component of the bi-exponential corresponds to the randomization of the fast subensemble's orientations, which leaves behind the decay of the slow subensemble's orientations. Each subensemble completely randomizes. This mechanism would require two distinct and well defined local structures that each give rise to a single exponential decay of its orientational anisotropy. Since the vast majority of the signal comes from the imidazolium head groups,(55) for this model to be true, there would have to be two imidazolium subensembles with distinctly different dynamics due to distinctly different environments. However, the volume fraction of water in the samples showing biexponential orientational decays is $\sim 5 \%$ or less. It seems physically unreasonable that such a small amount of water can perturb the system to the point of generating two distinct cation subensembles with dynamics that differ by two orders of magnitude. Such a scenario is physically unrealistic.

The alternative case is one in which there is a single ensemble that undergoes orientational relaxation with two timescales, one fast and restricted, and one slow and complete. This is known as wobbling-in-a-cone (WC).(55,114,115) In this model, the fast rotational diffusion component is restricted in a potential cone, while the cone itself rotationally diffuses on a slower time scale. The functional form of the correlation function is given by 


$$
C_{2}(t)=\left[S^{2}+\left(1-S^{2}\right) \exp \left(-t / \tau_{c}\right)\right] \exp \left(-t / \tau_{l}\right)
$$

where $\tau_{c}$ is the decay time of the diffusion within the cone, $\tau_{l}$ is the long decay time of complete diffusive reorientational relaxation, and $S$ is termed the order parameter.

Within this model, the fast decay time extracted with Equation $11, \tau_{1}$, is not the conerestricted decay time, but is related to it by

$$
\tau_{c}^{-1}=\tau_{1}^{-1}-\tau_{2}^{-1}
$$

and the cone semiangle, $\theta$, is obtained using

$$
S^{2}=\left[\frac{1}{2} \cos \theta(1+\cos \theta)\right]^{2} .
$$

$S^{2}$ obeys the inequality $0 \leq S^{2} \leq 1$, with $S^{2}=1$ corresponding to a cone semiangle of $0^{\circ}$ (no cone) and $S^{2}=0$ gives a cone semiangle of $180^{\circ}$ (unrestricted reorientation). $\tau_{l}$ in Equation 12 is $\tau_{1}$ in Equation 11. As can be seen from Equation 12, $S^{2}$ is the amplitude of the slow decay. While the diffusion constant of the final orientational randomization is given by $D_{l}=\left(6 \tau_{l}\right)^{-1}$, the diffusion constant of the cone-restricted motions $\left(D_{c}\right)$ depends on the cone angle and is given by the expression

$$
D_{c}=\frac{x^{2}\left(1+x_{c}\right)^{2}\left\{\ln \left[\left(1+x_{c}\right) / 2\right]+\left(1-x_{c}\right) / 2\right\}}{\tau_{c}\left(1-S^{2}\right)\left[2\left(x_{c}-1\right)\right]}+\frac{\left(1-x_{c}\right)\left(6+8 x_{c}-x_{c}^{2}-12 x_{c}^{3}-7 x_{c}^{4}\right)}{24 \tau_{c}\left(1-S^{2}\right)}
$$

where $x=\cos \theta$. Because of this complex dependence on $\theta$, it is not meaningful to directly compare $\tau_{c}$ values, but instead comparison was made of $D_{c}$, which therefore requires knowledge of $S^{2}$.(55) The WC model is often applied to systems in which the rotating species is tethered to a slow or immovable scaffold, such as a protein 
backbone(116) or a polymer chain.(117) The WC model was used to analyze the biexponential decays seen for the higher water content mixtures.(55)

A standard way to analyze the rotational diffusion times is through the DebyeStokes-Einstein (DSE) equation, Equation 2 in Section II. $(55,118)$ While the DSE equation is hydrodynamic, it works remarkably well for describing the rotational motion of molecules as was shown for the fluorescence depolarization experiments discussed in Section II.(119-121) In Equation 2, $V, f, C$, and $T$ are constants. Then, the orientational relaxation decay times, $\tau$, plotted versus viscosity $(\eta)$ should fall on a line if the system obeys the DSE equation, that is, the orientational relaxation is hydrodynamic.

All of the OHD-OKE exponential decay times from the fits are plotted versus viscosity in Figure 13. For all the RTILs studied here, the addition of water reduces the shear viscosity of the solution. As can be seen in Figure 13A, the long time scale exponential decays in the $\mathrm{BmImBF}_{4} /$ water samples show DSE behavior. As the viscosity of the solution decreases, due to the addition of water, the orientational decay time becomes correspondingly faster, with a value of $1.4 \mathrm{~ns}$ for the pure RTIL and $30 \mathrm{ps}$ for the most dilute solution $\left(1: 100 \mathrm{BmImBF}_{4}: \mathrm{H}_{2} \mathrm{O}\right)$. For the next longest chain RTIL, $\mathrm{HmImBF}_{4}$ (Figure 13B), the DSE relation is also obeyed for the slowest exponential, but now there is a second, faster exponential relaxation process seen in the 1:1 and watersaturated samples. These are the red circles in Figures 13B through D. The black circles in the figures come from analysis using the $\mathrm{WC}$ model discussed above and further below. (For a number of points, the black circle is on top of the red circle, and obscures it.) Figure 13C shows the DSE plot for OmImBF 4 . There is a fast exponential rotational diffusion time that emerges for the water-rich samples (3:1, 1:1, and water-saturated, red 
circles). At low water content, the $\mathrm{OmImBF}_{4}$ samples are fit to Equation 5 (single exponentials at long time) and display DSE behavior (Figure 13C, blue points at high viscosities). However, as the water content is increased, the long time decays become biexponential (see Figure 11C bottom curve). The fast component (red points) still has DSE dynamics, however, the slow component of the bi-exponential fits appears to plateau and turn up as water is added. Thus, while the viscosity is decreasing upon water addition, the slow rotational diffusion time is not affected in the water-rich samples in a manner consistent with the DSE equation.

This non-DSE behavior becomes much more dramatic in the $\mathrm{DmImBF}_{4}$ plots shown in Figure 12. Again, the rotation times for the water-poor $\mathrm{DmImBF}_{4}$ samples (blue circles, solid line) are single exponentials. The slow single exponential and the fast component of the water-rich samples (inset) are linear in viscosity. But the slow rotation time of the bi-exponential decays in the water-rich samples now moves in the opposite direction from that predicted by the DSE equation. As water is added the viscosity is going down, and yet the slow component of the rotational dynamics is drastically slowing down. For the water-saturated $\mathrm{DmImBF}_{4}$, the slow component is $89 \mathrm{ns,} \mathrm{while} \mathrm{the} \mathrm{fast}$ component is only $780 \mathrm{ps}$ (see Figure 12). The pure (no water) $\mathrm{DmImBF}_{4}$ has a long time single exponential rotational diffusion time of $14.3 \mathrm{~ns}$. The amplitude of the slow component grows upon addition of water and the concentration at which the biexponential behavior emerges shifts to lower water concentrations as the alkyl tail length increases.

In contrast to the pure RTILs, once a sufficient amount of water is added, the mixtures show distinctly bi-exponential OHD-OKE decays. The bi-exponential 
dynamics are interpret as a local stiffening of the cation's alkyl tail-tail associations.(55) The bi-exponential dynamics are particularly prominent in the water-saturated $\mathrm{DmImBF}_{4}$ sample in which a very pronounced separation of time scales is observed (see Figure 12). As discussed above, the fast component is attributed to the wobbling-in-a-cone motions of the tethered imidazolium head groups and the longest time component to slow, overall cation reorientation due to alkyl tail aggregates. The cone angle for the wobbling imidazolium head groups was determined for the highest water content $\mathrm{DmImBF}_{4}$ sample, which has the largest separation in time scales. Detailed analysis of the data yielded a cone angle of $41^{\circ} \pm 1^{\circ}$.(55) The local stiffening of the alkyl tail associations prevents orientational relaxation of the head groups and tails on a single time scale. As water is added, it will strongly interact with the ionic groups of the RTILs. Because there is insufficient water to fully hydrate the ions, the ions and water molecules will organize to maximize the water-ion interactions. This organization of the ionic regions forces a structure on the entangled alkyl tails that come together from different ion clusters. For the long alkyl chains, the chain arrangement "locks" them into stable configurations that resist randomization of their orientations. As water is added up to the saturation point for $\mathrm{OmImBF}_{4}$ and $\mathrm{DimImBF}_{4}$ in particular, the organization of the ionic regions increases, and the alky chain regions become increasingly resistant to orientational randomization. The result is the observed slowing of overall orientational relaxation (slowest OHD-OKE decay component) as water is added even though the viscosity of the solutions decreases. The constrained alkyl chain motions become so slow that the wobbling motion of the cation head groups occurs on a much faster time scale and is observable as a separate exponential decay (the faster exponential decay component). 
The details of the observed dynamics, that is, the bi-exponential decays at higher water contents, is consistent with the water-induced gelation seen in a few alkylmethylimidazolium RTILs,(47-49) but the solutions studied here phase separate before a gelation concentration is reached. Gelation of an RTIL is discussed in the next section. The local structuring and stiffening of the alkyl tail associations suggest that reaction rates in RTIL/water co-solvent mixtures should be highly dependent on the nature of the reactants and not solely on the global viscosity of the mixture.

\section{Dynamical Signature of the Approach Gelation in RTILs}

There are extensive reports of the aggregation behavior of RTILs in water, but most of these involve dilute RTILs in water or studies of RTILs that do not show gelation.(44,104,122-134) Studies of gelling systems have largely focused on the structure of the gel.(46-49,135-139) Dynamical measurements of gelling RTIL/water systems are limited in number and scope.(140-142) The OHD-OKE studies described above demonstrated that the orientational dynamics anomalously slowed down as water was added to the longer chain RTILs. The orientational relaxation (rotational diffusion) clearly becomes bi-exponential with the slow decay becoming slower even as the viscosity decreases with the addition of water. This behavior was attributed to organization of the ionic regions via ion-water interactions, which in turn cause long alkyl chains to arrange in a manner that resists orientational relaxation. The biexponential relaxation may be a dynamical signature of the approach to gelation.(55) $\mathrm{OmImBF}_{4}$ and $\mathrm{DimImBF}_{4}$ saturate at relatively low water contents. Presumably, if more water could be added, these RTILs would gel. 
However, $\mathrm{OmImCl}$ is completely miscible with water, and it gels. Figure 14 shows the dependence of the OmImCl viscosity with water content.(143) The viscosity decreases monotonically as water is added to the pure RTIL. However, in the range of water content from $\sim 2.2$ to $\sim 4.2$ water molecules per ion pair (mass fraction $\mathrm{H}_{2} \mathrm{O} \sim 0.16$ to $\sim 0.25$, mole fractions 0.69 to 0.81 ) the mixture becomes a gel. It has essentially infinite viscosity.

Some binary mixtures of pure RTILs show gelation behavior,(144) and some can be caused to gel with small molecule "gelators" to form what is now called an ionogel.(145,146) There is considerable interest in the RTILs that undergo gelation solely by the addition of water. $(47,135,136,147)$ Members of this latter group are probably better classified as lyotropic liquid crystals since many reports indicate formation of lamellar, cubic, hexagonal, and micellular phases as the water concentration is increased.(47,49,135) The fact that mixing water with some RTILs (i.e. mixing two liquids) can lead to stable gel formation may be unique to this class of liquids. Of those RTILs that show this hydrogel/ionogel behavior, $\mathrm{OmImCl}$ is particularly interesting. The gel has a melting temperature just above room temperature,(135) and $\mathrm{OmImCl}$ is the probably the smallest hydrogelator known.

We examined $\mathrm{OmImCl}$ in the identical manner that we used to study the dynamics of the alkylmethylimdazolium tetrafluoroborate samples.(56) Figure 15 displays the OHD-OKE data as a function of the water content. The numbers next to the data curves are the number of ion pairs to the number of water molecules (ion:water). The red dashed curves are the fits to the data (black curves) with Equation 5 using only the second and third power laws and the exponential when the final decay is single 
exponential or Equation 11 when the final decay is bi-exponential. (The curves have been offset along the vertical axis for clarity of presentation.) At the lowest water content, 22:1, the final decay is a single exponential, as was observed for $\mathrm{OmImBF}_{4}$. However, with $\mathrm{OmImCl}$ it is possible to go to very high water concentration. For the highest water content, $1: 100$, the final decay is also a single exponential. In contrast to the very high and low water contents, the OHD-OKE decays for intermediate water concentrations are distinctly bi-exponential, similar to the previous experiments on 1-alkyl-3methylimidazolium tetrafluoroborate/water mixtures.(55)

As in the treatment of the $\mathrm{OmImBF}_{4}$ and $\mathrm{DmImBF}_{4} /$ water mixtures, the OmImCl bi-exponential decays were analyzed with the wobbling-in-a-cone model in which the fast, cone-limited motions correspond to the imidazolium head group restricted reorientation and the slow motions to the complete orientational relaxation of the head group plus alkyl chain.(56) The extracted "relative" order parameters indicate that as the OmimCl/water gelling concentration is approached from the water poor side, the motions of the imidazolium head group become less restricted. In contrast, as the gelling region is approached from water rich side, the head group motions become more restricted. Analysis of the longer chain $\mathrm{C}_{\mathrm{n}} \mathrm{mimBF}_{4} /$ water data(55) indicates that as water is added to the pure RTIL the head group motions become more restricted.(56)

Figure 16 shows the slowest decay time constant (black circles) for the $\mathrm{OmImCl} /$ water mixtures as a function of viscosity (water content). The dashed line shows what the decay time constant vs. viscosity trend would be if the final complete orientational randomization was properly described in terms of hydrodynamics, that is, it obeyed the Debye-Stokes-Einstein equation (Equation 2). The blue shaded region 
indicates the viscosity range over which the system is a gel. Comparison of the data points and the dashed line confirms that the viscosity dependence is far from hydrodynamic. The overall cation reorientation time in the $\mathrm{OmimCl} /$ water samples is anomalously slow compared to the DSE equation calculation. The experiments cannot be conducted in the gel region because the sample is not transparent. However, data were taken very close to the gel region with the water contents just below (1:2) and just above (1:4.5) the water concentrations that produce gellation. On either side of the gel boundary, the long decay time constants (complete orientational randomization) are the same within experimental error even though the viscosity is different by a factor of somewhat greater than two. The results indicate that the bi-exponential decays are a precursor to the onset of gelation. The nature of the gel has been investigated. It has been suggested that the gel phase of $\mathrm{OmImCl}$ is dominated by a hexagonal structure.(135) Thus, the bi-exponential decays seen in the OHD-OKE studies may be associated with the organization of the imidazolium head groups and the alkyl chains that give rise to the large deviations from hydrodynamic behavior and are the precursor to the formation of an ordered gel phase.

\section{Concluding Remarks}

Time dependent experiments were employed to explicate aspects of the relationship between dynamics and structure in RTILs. The mesoscopic heterogeneity of RTILs was shown clearly by the distinct solvation environments of the non-polar fluorescent probe perylene and the charged fluorescent probe MPTS (see Figure 1). For the RTILs with sufficiently long alkyl chains, the friction coefficients obtained from the orientational relaxation of perylene in RTILs converged towards those of perylene in long 
chain length alkanes. The results show that the RTIL alkyl regions are truly distinct with properties that approach those of a pure long alkane chain. Chemical reactions or other processes that occur in the alkyl regions of a long chain RTIL may be independent of the overall bulk properties of the RTIL, such as viscosity. The ionic MPTS fluorophore was located in the ionic regions of the RTILs and displayed orientational dynamics consistent with very strong associative interactions between the negatively charged groups on the MPTS and the alkylmethylimidazolium cations. Lithium cations in the form of $\mathrm{Li}^{+}$ bis(trifluoromethanesulfonyl)imide ${ }^{-}$were added to the alkylmethylimidazolium bis(trifluoromethanesulfonyl)imide RTILs. The lithium cations locate in the ionic regions. The time dependent fluorescence depolarization experiments on perylene located in the alkyl regions showed that the addition of $\mathrm{Li}^{+}$in the ionic regions modified the structure of the alkyl chain. Although the RTILs are nanoheterogeneous with ionic and organic regions, the regions strongly influence each other.

In contrast to the fluorescence depolarization studies of the orientational relaxation of fluorophores selectively solvated in different regions of the RTILs, OHDOKE experiments were used to examine the bulk liquid orientational relaxation dynamics. The experiments on pure RTILs, which spanned many decades of time and signal amplitude, displayed a complex time dependence that consisted of several power law decays at short time followed by a final exponential decay (see Figure 7). The final exponential decay is associated with complete orientational randomization of the liquid while the power laws reflect the dynamics that occur from caging in which orientational relaxation of a molecule depends on the structural relaxation of surrounding molecules, the cage. The functional form of the RTIL data was shown to be identical to that found 
for many non-ionic non-polar organic liquids. The data were analyzed with schematic mode coupling theory. The RTIL data obeyed standard MCT scaling laws as do many non-ionic organic liquids (see Figure 10). The excellent fits with MCT and the applicability of the MCT scaling relationships shows that MCT provides a very good mathematical description of the RTIL bulk orientational relaxation. The important aspect of the usefulness of MCT to describe RTIL dynamics is that MCT is a theory of homogeneous liquids. Therefore, in terms of the bulk orientational dynamics of RTILs, there is no manifestation of the nanoscopic heterogeneous nature of the liquids. An RTIL can exhibit properties related to its nanoheterogeneity or behave as a simple liquid depending on the property of interest and the experimental observable.

The OHD-OKE experiments showed that the behavior of RTILs differed substantially from that of simple organic liquids when small amounts of water were added. For the short chain length alkylmethylimidazolium tetrafluoroborate RTILs, the final decay was a single exponential independent of the water content. The orientational relaxation times displayed hydrodynamic behavior, that is, they were linear in the viscosity. However, for the longer chain RTILs, particularly $\mathrm{OmImBF}_{4}$ and $\mathrm{DmImBF}_{4}$, the long time decay became bi-exponential at water concentrations near saturation. This behavior was most pronounced for $\mathrm{DmImBF}_{4}$ (see Figure 12). The final exponential decay deviated drastically from hydrodynamic behavior becoming slower as the viscosity decreased (see Figure 13D). This behavior was described in terms of the influence water has on the structure of the ionic regions. When the number of water molecules is small compared to the number necessary to fully solvate the individual cations and anions, the ions will organize to maximize their sharing of the water molecules, which in turn 
changes the structure of the alkyl regions. The long alkyl chains from different ionic regions effectively become locked into very stable configurations that resist structural rearrangement, i. e., orientational relaxation. The fast component of the bi-exponential decay arises from wobbling of the cations in a limited cone of angles with essentially fixed alkyl chain structure. On a much longer time scale, complete randomization of the entire liquid structure occurs, giving rise to the long component of the bi-exponential decay.

With $\mathrm{BF}_{4}{ }^{-}$as the anion, only a relatively small amount of water can be added to the long chain length RTILs before the miscibility limit is reached. Some RTILs form gels over a narrow range of water concentrations. The dramatic slowing of the structural dynamics of $\mathrm{DmImBF}_{4}$ as water was added raised the interesting question if the slowing and bi-exponential dynamics are signatures of the approach to gel formation. $\mathrm{OmImCl}$ is infinitely miscible with water and forms a gel over a small range of water concentrations, i. e., about 2.2 to 4.2 water molecules per ion pair. The OHD-OKE data showed that the slowest dynamics were single exponential decays for either exceedingly little water or a great deal of water. However, at intermediate water concentrations, the decays became bi-exponential (see Figure 15) and did not display hydrodynamic behavior (see Figure 16). In addition, the decay times for complete orientational relaxation were the same just below and just above the gel region (see Figure 16) even though the viscosities were quite different. The results indicated that the non-hydrodynamic behavior and biexponential dynamics are signatures of the approach to the gel transition.

Structure and dynamics play off against each other. The use of RTILs in applications requires understanding both structure and dynamics. The methods discussed 
here are being extended by the application of ultrafast 2D IR vibrational echo experiments to the study of RTILs.(148,149) The 2D IR experiments provide a different type of observable and have the potential to greatly increase our understanding of RTIL dynamics and structure.

\section{Acknowledgements}

I would like to thank the many members of the Fayer Group who contributed to this research over many years. The research presented here was supported by the Division of Chemical Sciences, Geosciences, and Biosciences, Office of Basic Energy Sciences of the U.S. Department of Energy through Grant \# DE-FG03-84ER13251, the Division of Chemistry, Directorate of Mathematical and Physical Sciences, National Science Foundation Grant \# CHE-1157772, and the Air Force Office of Scientific Research Grant \# FA9550-12-1-0050.

\section{Biography - Michael D. Fayer}

Michael D. Fayer is the David Mulvane Ehrsam and Edward Curtis Franklin Professor of Chemistry at Stanford University. Fayer received his Ph.D. in chemistry from the University of California at Berkeley in 1974. He went directly to Stanford, where he has been a faculty member for forty years. Fayer is a member of the National Academy of Sciences, and has won a number of awards for his work developing and applying ultrafast laser techniques to the study of the dynamics of complex molecular systems. He has published two books, "Elements of Quantum Mechanics," Oxford University Press (2001), a graduate level text book, and "Absolutely Small - How Quantum Theory 
Explains Our Everyday World," AMACOM (2010), a quantum book for laymen, high school and undergraduate students, which explains quantum theory, atoms, and molecules with no math. 


\section{References}

(1) S A Forsyth, J M Pringle, D R MacFarlane, Aust. J. Chem. 57 (2004) 113-119.

(2) W J Li, Z F Zhang, B X Han, S Q Hu, Y Xie, G Y Yang, J. Phys. Chem. B 111 (2007) 6452-6456.

(3) M Sureshkumar, C-K Lee, Journal of Molecular Catalysis B: Enzymatic 60 (2009) 1-12.

(4) A Paczal, A Kotschy, Monatsh Chem 138 (2007) 1115-1123.

(5) J R Harjani, P U Naik, S J Nara, M M Salunkhe, Curr Org Synth 4 (2007) 354369.

(6) R Hagiwara, J S Lee, Electrochemistry 75 (2007) 23-34.

(7) S M Urahata, M C C Ribeiro, J. Chem. Phys. 120 (2004) 1855-1863.

(8) Y Wang, G A Voth, J. Am. Chem. Soc. 127 (2005) 12192-12193.

(9) B L Bhargava, R Devane, M L Klein, S Balasubramanian, Soft Matter 3 (2007) 1395-1400.

(10) J N Canongia Lopes, A a H Pádua, J. Phys. Chem. B 110 (2006) 3330-3335.

(11) M F C Gomes, J N C Lopes, A A H Padua, Top. Curr. Chem. 290 (2009) 161-183.

(12) O Russina, A Triolo, L Gontrani, R Caminiti, D Xiao, L G J Hines, R A Bartsch, E L Quitevis, N Pleckhova, K R Seddon, J. Phys.: Condens. Matter 21 (2009) 424121.

(13) A Triolo, O Russina, H J Bleif, E Di Cola, J. Phys. Chem. B 111 (2007) 46414644.

(14) A Triolo, O Russina, B Fazio, R Triolo, E Di Cola, Chem. Phys. Lett. 457 (2008) $362-365$. 
(15) A Sarkar, S Trivedi, G A Baker, S Pandey, J. Phys. Chem. B 112 (2008) 1492714936.

(16) J Guo, G a Baker, P C Hillesheim, S Dai, R W Shaw, S M Mahurin, Phys. Chem. Chem. Phys. 13 (2011) 12395-12398.

(17) A M Funston, T A Fadeeva, J F Wishart, E W Castner, Jr., J. Phys. Chem. B 111 (2007) 4963-4977.

(18) D Xiao, J R Rajian, A Cady, S Li, R A Bartsch, E L Quitevis, J. Phys. Chem. B 111 (2007) 4669-4677.

(19) D Xiao, J R Rajian, L G Hines, S Li, R A Bartsch, E L Quitevis, J. Phys. Chem. B $112(2008)$ 13316-13325.

(20) D Xiao, J R Rajian, S Li, R A Bartsch, E L Quitevis, J. Phys. Chem. B 110 (2006) 16174-16178.

(21) D A Turton, J Hunger, A Stoppa, G Hefter, A Thoman, M Walther, R Buchner, K Wynne, J. Am. Chem. Soc. 131 (2009) 11140-11146.

(22) P Yang, G a Voth, D Xiao, L G Hines, R a Bartsch, E L Quitevis, J. Chem. Phys. 135 (2011) 034502-034502.

(23) M M Mizoshiri, M., T Nagao, Y Mizoguchi, M Yao, J. Chem. Phys. 132 (2010).

(24) M Imanari, K I Uchida, K Miyano, H Seki, K Nishikawa, Phys. Chem. Chem. Phys. 12 (2010) 2959-2967.

(25) C Chiappe, Monatsh Chem 138 (2007) 1035-1043.

(26) R Atkin, G G Warr, J. Phys. Chem. B 112 (2008) 4164-4166.

(27) C Hardacre, J D Holbrey, C L Mullan, T G A Youngs, D T Bowron, J. Chem. Phys. 133 (2010) 074510. 
(28) H V R Annapureddy, H K Kashyap, P M De Biase, C J Margulis, J. Phys. Chem. B 114 (2010) 16838-16846.

(29) O Russina, A Triolo, Faraday Discuss. 154 (2011) 97-109.

(30) B Aoun, A Goldbach, M A Gonzalez, S Kohara, D L Price, M L Saboungi, J. Chem. Phys. 134 (2011) 104509.

(31) M Macchiagodena, L Gontrani, F Ramondo, A Triolo, R Caminiti, J. Chem. Phys. 134 (2011).

(32) H K Kashyap, C S Santos, H V R Annapureddy, N S Murthy, C J Margulis, E W Castner, Faraday Discuss. (2011).

(33) C S Santos, N S Murthy, G A Baker, E W Castner, J. Chem. Phys. 134 (2011).

(34) C S Santos, H V R Annapureddy, N S Murthy, H K Kashyap, E W Castner, C J Margulis, J. Chem. Phys. 134 (2011).

(35) K Fruchey, C M Lawler, M D Fayer, J Phys Chem B 116 (2012) 3054-3064.

(36) K Fruchey, M D Fayer, J. Phys. Chem. B 114 (2010) 2840-2845.

(37) H Jin, X Li, M Maroncelli, J. Phys. Chem. B 111 (2007) 13473-13478.

(38) S Tiwari, N Khupse, A Kumar, J. Org. Chem. 73 (2008) 9075-9083.

(39) E W Castner, C J Margulis, M Maroncelli, J F Wishart, Annual Review of Physical Chemistry, Vol 6262 (2011) 85-105.

(40) G Douhéret, J C R Reis, M I Davis, I J Fjellanger, H Høiland, Phys. Chem. Chem. Phys. 6 (2004) 784-792.

(41) J Dupont, R F de Souza, P A Z Suarez, Chem. Rev. 102 (2002) 3667-3691.

(42) N D Khupse, A Kumar, J. Phys. Chem. A 115 (2011) 10211-10217.

(43) N D Khupse, A Kumar, J. Solution Chem. 38 (2009) 589-600. 
(44) L A S Ries, F A do Amaral, K Matos, E M A Martini, M O de Souza, R F de Souza, Polyhedron 27 (2008) 3287-3293.

(45) S Feng, G A Voth, Fluid Phase Equilib. 294 (2010) 148-156.

(46) W Jiang, Y T Wang, G A Voth, J. Phys. Chem. B 111 (2007) 4812-4818.

(47) M A Firestone, P G Rickert, S Seifert, M L Dietz, Inorg. Chim. Acta 357 (2004) 3991-3998.

(48) J Bowers, C P Butts, P J Martin, M C Vergara-Gutierrez, R K Heenan, Langmuir 20 (2004) 2191-2198.

(49) B L Bhargava, M L Klein, Mol. Phys. 107 (2009) 393-401.

(50) C Lawler, M D Fayer, J. Phys. Chem. B 117 (2013) 9768-9774.

(51) H Cang, J Li, M D Fayer, J. Chem. Phys. 119 (2003) 13017-13023.

(52) J Li, I Wang, K Fruchey, M D Fayer, J. Phys. Chem. A 110 (2006) 10384-10391.

(53) D D Brace, S D Gottke, H Cang, M D Fayer, J. Chem. Phys. 116 (2002) 15981606.

(54) H Cang, J Li, H C Andersen, M D Fayer, J. Chem. Phys. 123 (2005) 064508

(55) A L Sturlaugson, K S Fruchey, M D Fayer, J. Phys. Chem. B 116 (2012) 17771787.

(56) A L Sturlaugson, A A Arima, H E Bailey, M D Fayer, J. Phys. Chem. B 117 (2013) 14775-14784.

(57) J A Ingram, R S Moog, N Ito, R Biswas, M Maroncelli, J. Phys. Chem. B 107 (2003) 5926-5932.

(58) K S Mali, G B Dutt, T Mukherjee, J. Chem. Phys. 128 (2008).

(59) N Ito, S Arzhantsev, M Maroncelli, Chem. Phys. Lett. 396 (2004) 83-91. 
(60) T J Chuang, Eisentha.Kb, J. Chem. Phys. 57 (1972) 5094-\&.

(61) F Perrin, J. Phys. et Rad. 7 (1936) 1-11.

(62) R J Sension, R M Hochstrasser, J. Chem. Phys. 98 (1993) 2490-2490.

(63) G K Youngren, A Acrivos, J. Chem. Phys. 63 (1975) 3846-3848.

(64) J Xu, X Shen, J R Knutson, J. Phys. Chem. A 107 (2003) 8383-8387.

(65) Y R Kim, R M Hochstrasser, J. Phys. Chem. 96 (1992) 9595-9597.

(66) J R Mannekutla, P Ramamurthy, B G Mulimani, S R Inamdar, Chem. Phys 340 (2007) 149-157.

(67) M J Wirth, S H Chou, J. Phys. Chem. 95 (1991) 1786-1789.

(68) S W Pauls, J F Hedstrom, C K Johnson, J. Chem. Phys. 237 (1998) 205-222.

(69) Y Jiang, G J Blanchard, J Phys Chem 98 (1994) 6436-6440.

(70) T Troxler, J R Stratton, P G Smith, M R Topp, J. Chem. Phys. 101 (1994) 92199231.

(71) A Chaumont, G Wipff, Journal of Molecular Liquids 131 (2007) 36-47.

(72) A A H Pádua, M F Costa Gomes, J N A Canongia Lopes, Acc. Chem. Res. 40 (2007) 1087-1096.

(73) D Xiao, L G Hines, S F Li, R A Bartsch, E L Quitevis, O Russina, A Triolo, J. Phys. Chem. B 113 (2009) 6426-6433.

(74) L A Blanchard, Z Gu, J F Brennecke, J. Phys. Chem. B 105 (2001) 2437-2444.

(75) K G Spears, L E Cramer, J. Chem. Phys. 30 (1978) 1-8.

(76) T-W Nee, R Zwanzig, J. Chem. Phys. 52 (1970) 6353-6363.

(77) M-L Horng, J A Gardecki, M Maroncelli, J. Phys. Chem. A 101 (1997) 10301047. 
(78) A Takada, K Imaichi, T Kagawa, Y Takahashi, J. Phys. Chem. B 112 (2008) 9660-9662.

(79) J A Vega, J Zhou, P A Kohl, Journal of The Electrochemical Society 156 (2009) A253-A259.

(80) S Duluard, J Grondin, J-L Bruneel, I Pianet, A Grélard, G Campet, M-H Delville, J-C Lassègues, Journal of Raman Spectroscopy 39 (2008) 627-632.

(81) M J Monteiro, F F C Bazito, L J A Siqueira, M C C Ribeiro, R M Torresi, J. Phys. Chem. B 112 (2008) 2102-2109.

(82) Y Umebayashi, H Hamano, S Seki, B Minofar, K Fujii, K Hayamizu, S Tsuzuki, Y Kameda, S Kohara, M Watanabe, J. Phys. Chem. B 115 (2011) 12179-12191.

(83) B G Nicolau, A Sturlaugson, K Fruchey, M C C Ribeiro, M D Fayer, J. Phys. Chem. B 114 (2010) 8350-8356.

(84) O Borodin, G D Smith, W Henderson, J. Phys. Chem. B 110 (2006) 16879-16886.

(85) M Galiński, A Lewandowski, I Stępniak, Electrochimica Acta 51 (2006) 55675580.

(86) M Fury, J Jonas, J. Chem. Phys. 65 (1976) 2206-2210.

(87) C Hu, R Zwanzig, J. Chem. Phys. 60 (1974) 4354-4357.

(88) D McMorrow, W T Lotshaw, G A Kenney-Wallace, IEEE J. Quant. Elec. 24 (1988) 443-454.

(89) G Hinze, D D Brace, S D Gottke, M D Fayer, J. Chem. Phys. 113 (2000) 37233733.

(90) J P Boon, S Yip, Molecule Hydrodynamics, McGraw-Hill, New York, 1980.

(91) Y Kai, S Kinoshita, M Yamaguchi, T Yagi, J. Mol. Liquids 65-6 (1995) 413-416. 
(92) Y X Yan, K A Nelson, J. Chem. Phys. 87 (1987) 6240-6256.

(93) Y X Yan, K A Nelson, J. Chem. Phys. 87 (1987) 6240.

(94) F W Deeg, J J Stankus, S R Greenfield, V J Newell, M D Fayer, J. Chem. Phys. 90 (1989) 6893-6902.

(95) Y X Yan, L G Cheng, K A Nelson, Adv. Infrared and Raman Spectrosc. 16 (1987) 299.

(96) S D Gottke, D D Brace, G Hinze, M D Fayer, J. Phys. Chem. B 105 (2001) 238245.

(97) S D Gottke, Stanford University, Stanford, 2002.

(98) H Cang, V N Novikov, M D Fayer, J. Chem. Phys. 118 (2003) 2800-2807.

(99) H Cang, V N Novikov, M D Fayer, Phys. Rev. Lett. 90 (2003) 197401(197404).

(100) W Götze, Liquids, Freezing and Glass Transition, Elsevier Science Publishers, Amsterdam, 1989.

(101) W Gotze, L Sjogren, Rep. Prog. Phys. 55 (1992) 241-376.

(102) A L Zhu, J J Wang, L J Han, M H Fan, Chem. Eng. J. 147 (2009) 27-35.

(103) I Bou Malham, M Turmine, J. Chem. Thermodyn. 40 (2008) 718-723.

(104) E Rilo, J Vila, J Pico, S Garcia-Garabal, L Segade, L M Varela, O Cabeza, J. Chem. Eng. Data 55 (2010) 639-644.

(105) K R Seddon, A Stark, M J Torres, Clean Solvents, 2002, p. 34-49.

(106) A Arce, H Rodriguez, A Soto, Fluid Phase Equilib. 242 (2006) 164-168.

(107) K R Harris, M Kanakubo, L A Woolf, J. Chem. Eng. Data 51 (2006) 1161-1167.

(108) B Mokhtarani, M M Mojtahedi, H R Mortaheb, M Mafi, F Yazdani, F Sadeghian, J. Chem. Eng. Data 53 (2008) 677-682. 
(109) L G Sanchez, J R Espel, F Onink, G W Meindersma, A B de Haan, J. Chem. Eng. Data 54 (2009) 2803-2812.

(110) J Restolho, A P Serro, J L Mata, B Saramago, J. Chem. Eng. Data 54 (2009) 950955.

(111) M G Freire, C M S S Neves, I M Marrucho, J A P Coutinho, A M Fernandes, J. Phys. Chem. A 114 (2010) 3744-3749.

(112) H Cang, J Li, V N Novikov, M D Fayer, J. Chem. Phys. 118 (2003) 9303-9311.

(113) A L Sturlaugson, K S Fruchey, S R Lynch, S R Aragon, M D Fayer, J. Phys. Chem. B 114 (2010) 5350-5358.

(114) G Lipari, A Szabo, Biophys. J. 30 (1980) 489-506.

(115) C C Wang, R Pecora, J. Chem. Phys. 72 (1980) 5333-5340.

(116) K Sahu, S K Mondal, S Ghosh, D Roy, K Bhattacharyya, J. Chem. Phys. 124 (2006).

(117) P B Leezenberg, A H Marcus, C W Frank, M D Fayer, J Phys Chem 100 (1996) 7646-7655.

(118) D Kivelson, in: T. Dorfmüller, R. Pecora (Eds.), Rotational Dynamics of Small and Macromolecules, Springer, Berlin, 1987.

(119) G R Alms, D R Bauer, J I Brauman, R Pecora, J. Chem. Phys. 58 (1973) 55705578.

(120) D R Bauer, G R Alms, J I Brauman, R Pecora, J. Chem. Phys. 61 (1974) 22552261.

(121) G R Alms, D R Bauer, J I Brauman, R Pecora, J. Chem. Phys. 59 (1973) 53105320. 
(122) S Dorbritz, W Ruth, U Kragl, Adv. Synth. Catal. 347 (2005) 1273-1279.

(123) L Almasy, M Turmine, A Perera, J. Phys. Chem. B 112 (2008) 2382-2387.

(124) R Vanyur, L Biczok, Z Miskolczy, Colloids Surf. A-Physicochem. Eng. Aspects 299 (2007) 256-261.

(125) T Takamuku, Y Kyoshoin, T Shimomura, S Kittaka, T Yamaguchi, J. Phys. Chem. B 113 (2009) 10817-10824.

(126) T Masaki, K Nishikawa, H Shirota, J. Phys. Chem. B 114 (2010) 6323-6331.

(127) Y Danten, M I Cabaco, M Besnard, J. Phys. Chem. A 113 (2009) 2873-2889.

(128) J Luczak, J Hupka, J Thoming, C Jungnickel, Colloids Surf. A-Physicochem. Eng. Aspects 329 (2008) 125-133.

(129) H C Chang, J C Jiang, Y C Liou, C H Hung, T Y Lai, S H Lin, J. Chem. Phys. $129(2008)$.

(130) Z Miskolczy, K Sebok-Nagy, L Biczok, S Gokturk, Chem. Phys. Lett. 400 (2004) 296-300.

(131) B Fazio, A Triolo, G Di Marco, Journal of Raman Spectroscopy 39 (2008) 233237.

(132) H Shirota, R Biswas, J. Phys. Chem. B 116 (2012) 13765-13773.

(133) N A Smirnova, E A Safonova, Colloid J. 74 (2012) 254-265.

(134) C Schroder, T Rudas, G Neumayr, S Benkner, O Steinhauser, J. Chem. Phys. 127 (2007).

(135) I Goodchild, L Collier, S L Millar, I Prokes, J C D Lord, C P Butts, J Bowers, J R P Webster, R K Heenan, J. Colloid Interface Sci. 307 (2007) 455-468. 
(136) M A Firestone, J A Dzielawa, P Zapol, L A Curtiss, S Seifert, M L Dietz, Langmuir 18 (2002) 7258-7260.

(137) J C Ribot, C Guerrero-Sanchez, R Hoogenboom, U S Schubert, J. Mater. Chem. $20(2010) 8279-8284$.

(138) T Inoue, B Dong, L Q Zheng, J. Colloid Interface Sci. 307 (2007) 578-581.

(139) G D Zhang, X Chen, Y R Zhao, Y Z Xie, H Y Qiu, J. Phys. Chem. B 111 (2007) 11708-11713.

(140) T Mendez-Morales, J Carrete, O Cabeza, L J Gallego, L M Varela, J. Phys. Chem. B 115 (2011) 6995-7008.

(141) V Balevicius, Z Gdaniec, K Aidas, J Tamuliene, J. Phys. Chem. A 114 (2010) $5365-5371$.

(142) H V Spohr, G N Patey, J. Chem. Phys. 132 (2010).

(143) E Gomez, B Gonzalez, A Dominguez, E Tojo, J Tojo, J. Chem. Eng. Data 51 (2006) 696-701.

(144) J Kagimoto, N Nakamura, T Kato, H Ohno, Chem. Commun. (2009) 2405-2407.

(145) J Le Bideau, L Viau, A Vioux, Chem. Soc. Rev. 40 (2011) 907-925.

(146) K Hanabusa, H Fukui, M Suzuki, H Shirai, Langmuir 21 (2005) 10383-10390.

(147) J C Ribot, C Guerrero-Sanchez, R Hoogenboom, U S Schubert, Chem. Commun. 46 (2010) 6971-6973.

(148) D B Wong, C H Giammanco, E E Fenn, M D Fayer, J. Phys. Chem. B 117 (2013) 623-635.

(149) Z Ren, A S Ivanova, D Couchot-Vore, S Garrett-Roe, J. Phys. Chem. Lett. 5 (2014) 1541-1546. 
Table 1 - Perylene exp. frictional coefficients, $C$.

\begin{tabular}{|l|l|}
\hline Solvent & Friction Coefficient \\
\hline $\mathrm{C}_{2} \mathrm{mIm}^{+}$ & $0.0858 \pm 0.008$ \\
\hline $\mathrm{C}_{4} \mathrm{mIm}^{+}$ & $0.0799 \pm 0.002$ \\
\hline $\mathrm{C}_{6} \mathrm{mIm}^{+}$ & $0.0733 \pm 0.001$ \\
\hline $\mathrm{C}_{8} \mathrm{mIm}^{+}$ & $0.0583 \pm 0.0007$ \\
\hline paraffin oil & $0.0483 \pm 0.001$ \\
\hline Theory Slip & 0.085 \\
\hline
\end{tabular}

Table 2 - Ratios of in and out of plane experimental friction coefficients to their theoretical slip values.

\begin{tabular}{|l|c|c|}
\hline & in-plane & out of plane \\
\hline $\mathrm{C}_{2} \mathrm{mIm}^{+}$ & 1.2 & 0.39 \\
\hline $\mathrm{C}_{4} \mathrm{mIm}^{+}$ & 1.0 & 0.44 \\
\hline $\mathrm{C}_{6} \mathrm{mIm}^{+}$ & 0.90 & 0.53 \\
\hline $\mathrm{C}_{8} \mathrm{mIm}^{+}$ & 0.67 & 0.54 \\
\hline paraffin oil & 0.55 & 0.60 \\
\hline
\end{tabular}

Table 3 - MPTS exp. friction coefficients $C$.

\begin{tabular}{|l|c|}
\hline Solvent & Friction Coefficient \\
\hline $\mathrm{C}_{2} \mathrm{mIm}^{+}$ & $2.22 \pm 0.05$ \\
\hline $\mathrm{C}_{4} \mathrm{mIm}^{+}$ & $2.36 \pm 0.03$ \\
\hline $\mathrm{C}_{6} \mathrm{mIm}^{+}$ & $2.77 \pm 0.04$ \\
\hline Water & $0.99 \pm 0.02$ \\
\hline DMSO & $1.25 \pm 0.01$ \\
\hline
\end{tabular}




\section{Figure Captions}

Figure 1. Dimensions and coordinate system for perylene (A) and MPTS (B). The out of plane thickness is labeled $\mathrm{t}$.

Figure 2. Orientational relaxation times, $\tau=1 / 6 D(D$ is the average orientational diffusion coefficient) for perylene in five solvents plotted as a function of the viscosity ( $\eta$ ) divided by $k T$ (circles). The lines through the data points are fits using linear least squares. For clarity, the data sets have been shifted up $0.4 \mathrm{~ns}$ for $\mathrm{C}_{2} \mathrm{mIm}^{+}, 0.3 \mathrm{~ns}$ for $\mathrm{C}_{4} \mathrm{mIm}^{+}, 0.2 \mathrm{~ns}$ for $\mathrm{C}_{6} \mathrm{mIm}^{+}$, and $0.1 \mathrm{~ns}$ for $\mathrm{C}_{8} \mathrm{mIm}^{+}$.

Figure 3. Orientational relaxation times, $\tau=1 / 6 D(D$ is the orientational diffusion coefficient) for MPTS in three RTILs (A) and in two other solvents (B) plotted as a function of the viscosity $(\eta)$ divided by $k T$ (symbols). The lines through the data points are fits using linear least squares. For clarity, the $\mathrm{C}_{2} \mathrm{mIm}^{+}$and $\mathrm{C}_{4} \mathrm{mIm}^{+}$data have been shifted up 4ns and 2ns, respectively. Note the differences in the axes for A and B.

Figure 4. A. Orientational diffusion constants for perylene in $[\mathrm{BmIm}]\left[\mathrm{Tf}_{2} \mathrm{~N}\right]$ solutions at 298K with various $\mathrm{LiTf}_{2} \mathrm{~N}$ mole fractions. B. Temperature-dependent viscosities of $[\mathrm{BmIm}]\left[\mathrm{Tf}_{2} \mathrm{~N}\right]$ solutions with $\chi\left(\mathrm{LiTf}_{2} \mathrm{~N}\right)=0$ to 0.4 . The $\mathrm{y}$-axis is presented on a natural $\log$ scale. Lines connecting the data points are single exponential fits to the data.

Figure 5. Orientational relaxation times, $\tau=1 / 6 \mathrm{D}$, (symbols) for perylene plotted against the dynamic viscosity $\eta$ over $k T$, for $\chi\left(\operatorname{LiTf}_{2} \mathrm{~N}\right)=0$ to 0.4 . The plots are scaled and shifted vertically by the values given in the inset to aid visual comparison. The slopes of the plots have been preserved in each case. The lines through the data points are linear least-squares fits to the data and show that the orientational diffusion is hydrodynamic. 
Figure 6. The in-plane and out-of-plane friction coefficient ratios as a function of lithium cation concentration.

Figure 7. Optical heterodyned detected optical Kerr effect data for A an organic liquid dibutylphthalate and B the RTIL BmImBF 4 . Both types of liquids have the same functional form of their decays, i.e., two power laws followed by an exponential decay. Figure 8. OHD-OKE data for PMPIm at $T=233 \mathrm{~K}$ on logarithmic scale. The dashed line through the data is the fit using Equation 5. The two straight lines are aids to eyes indicating the intermediate power law and von Schweidler power law. The fast $\beta$ process power law can be seen at the shortest times. The inset shows the exponential decay (the $\alpha$ relaxation) at long times on a semi-logarithmic scale.

Figure 9. Temperature dependent OHD-OKE PMPIm data on a log plot. The data sets have been off-set along the vertical axis for clarity of presentation. Fits to the data using Equation 4 are also shown (dashed red curves).

Figure 10. Figure $10 \mathrm{~A}$ and $\mathrm{B}$ show data for the MCT scaling relationships for $\mathrm{A}$ the exponential decay time $\tau_{\alpha}$ and B the amplitude of the von Schweidler power law $d$. Both relationships only depend on the value of the von Schweidler power law exponent. Both sets of points fall on lines showing that the MCT scaling relationships are obeyed. The lines extrapolate to the MCT critical temperature, $T_{c}=231 \mathrm{~K}$.

Figure 11. OHD-OKE decays for 3 of the samples (solid black curves) and their fits to Equation 5 or 11 (dashed red curves). Curves have been offset along the vertical axis for clarity. A. $\mathrm{BmImBF}_{4}: \mathrm{H}_{2} \mathrm{O}$ samples. B. $\mathrm{HmImBF}_{4}: \mathrm{H}_{2} \mathrm{O}$ samples. C. $\mathrm{OmImBF}_{4}: \mathrm{H}_{2} \mathrm{O}$ samples. The bi-exponential decays are indicated by the arrows. 
Figure 12. OHD-OKE decays for 3 select $\mathrm{DmImBF}_{4}: \mathrm{H}_{2} \mathrm{O}$ samples (solid black curves) and their fits to Equation 5 or 11 (dashed red curves). Curves have been offset along the vertical axis for clarity. The inset shows the long time portion of the water-saturated $\mathrm{DmImBF}_{4}$.

Figure 13. DSE plots for $\mathrm{BmImBF}_{4}(\mathrm{~A}), \mathrm{HmImBF}_{4}(\mathrm{~B}), \mathrm{OmImBF}_{4}(\mathrm{C})$, and $\mathrm{DmImBF}_{4}$ (D). The inset in $\mathrm{D}$ is an expanded plot of the fast decay time constants. Color coding is as follows: $\tau_{2}$ - blue, slow component; $\tau_{1}$ - red, fast component; black, from wobblingin-a-cone model. Some of the red and black points are on top of each other. The lines are guides to the eye.

Figure 14. Viscosity vs. the mass fraction of water in OmImCl. The shaded blue area shows the range of water content in which the $\mathrm{OmImCl} /$ water mixtures form gels.

Figure 15. OHD-OKE decays for the OmimCl/water system for various water concentrations (solid black curves) and fits to Equations 5 or 11 (dashed red curves). Note the logarithmic axes. The curves have been offset along the vertical axis for clarity of presentation. Stable gel formation occurs between the OmimCl: water $=1: 2$ and 1:4.5 samples.

Figure 16. Hydrodynamic (DSE equation) type plot for the longest, overall cation complete orientational randomization decay times, $\tau_{l}$. The dashed black line inticates what the decays times would be if the system displayed hydrodynamic behavior (DSE Equation 2) as calculated using the maximum $\tau_{l}$ value. The concentration range over which the stable gel forms is shown in blue. 


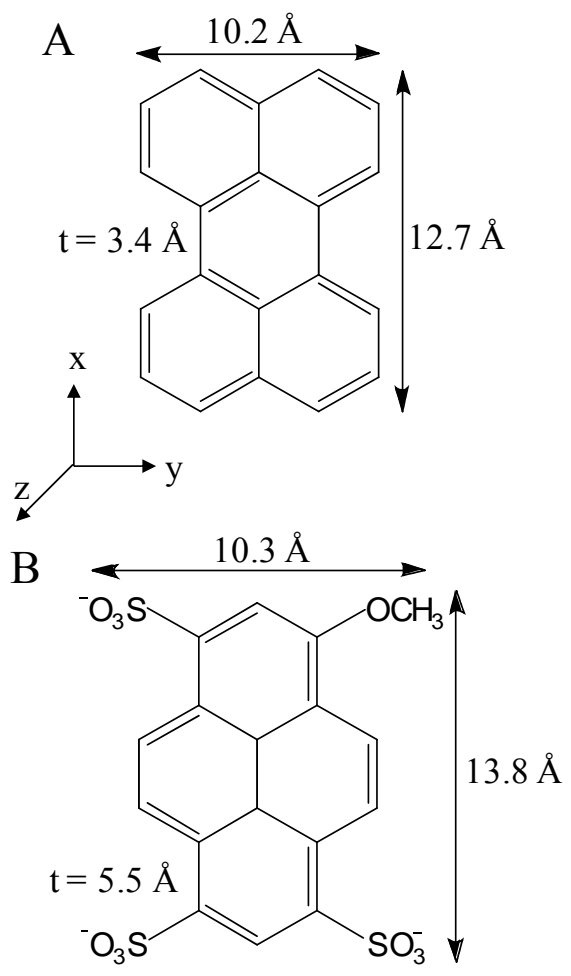

figure 1 


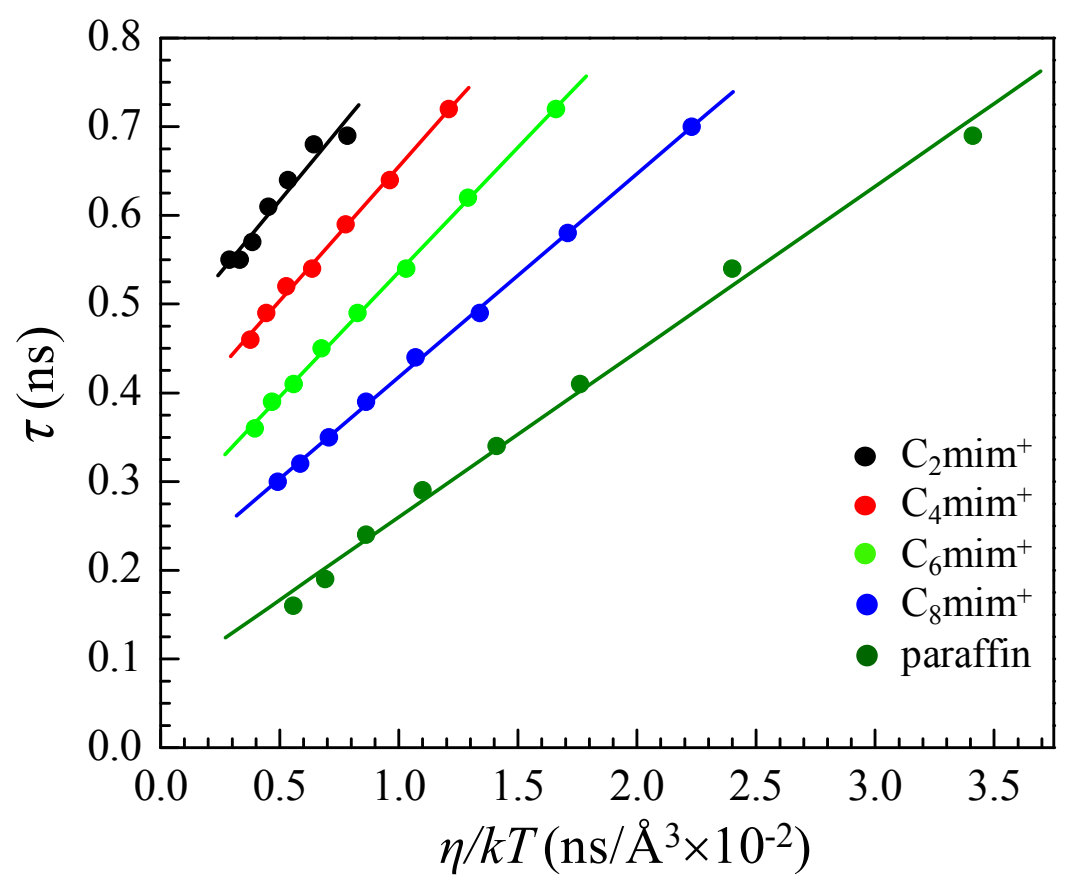

figure 2

29

30

31

32

33

34

35

36

37

38

39

40

41

42

43

44

45

46

47

48

49

50

51

52

53

54

55

56

57

58

59

60

61

62 

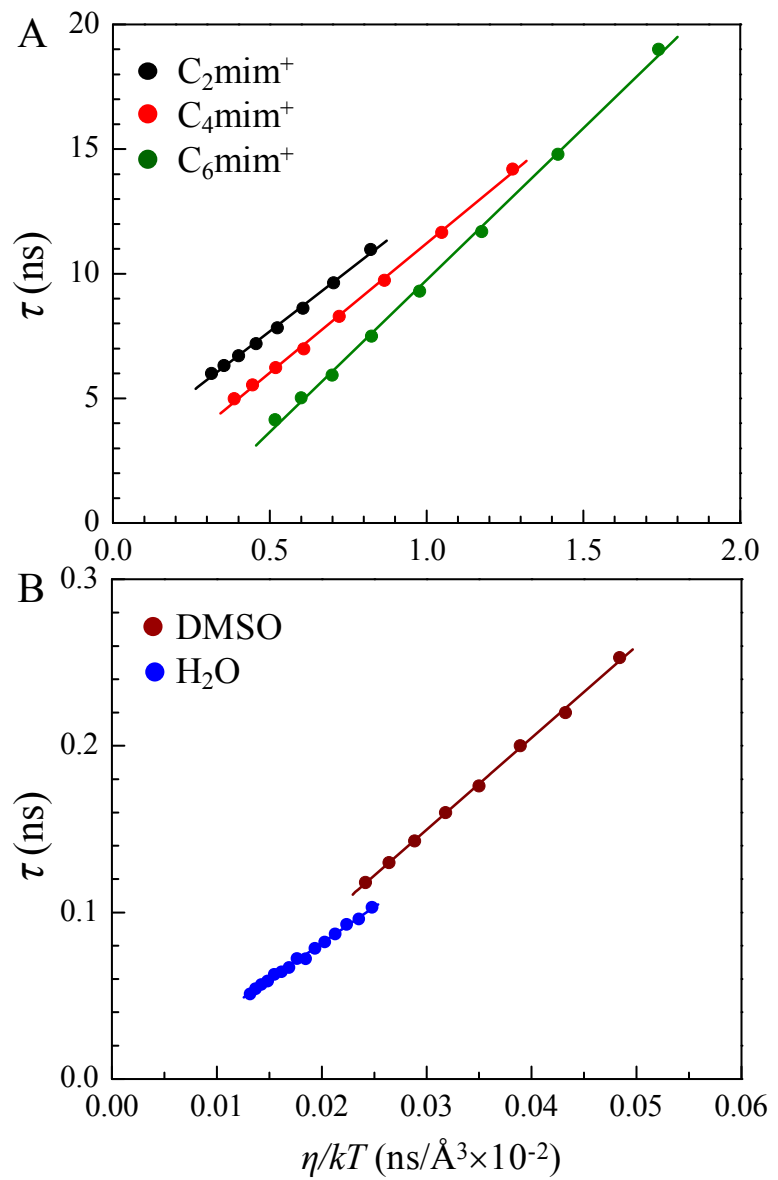

figure 3

39

40

41

42

43

44

45

46

47

48

49

50

51

52

53

54

55

56

57

58

59

60

61

62 

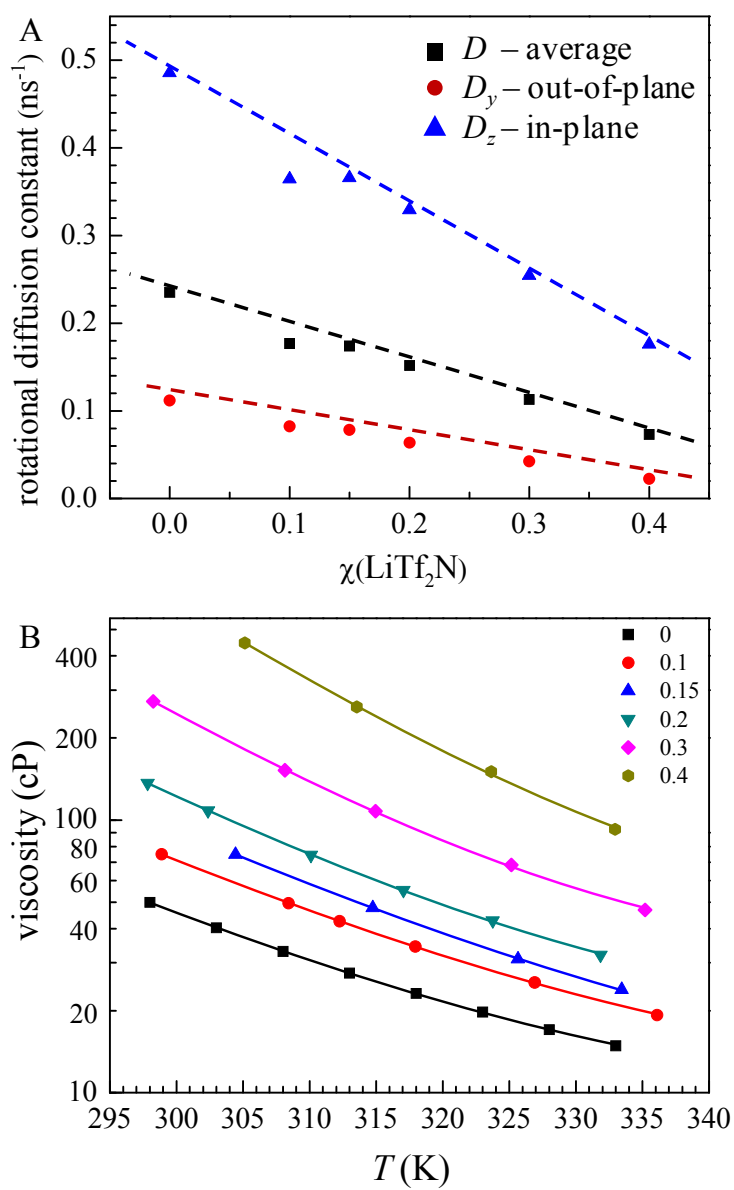

figure 4 


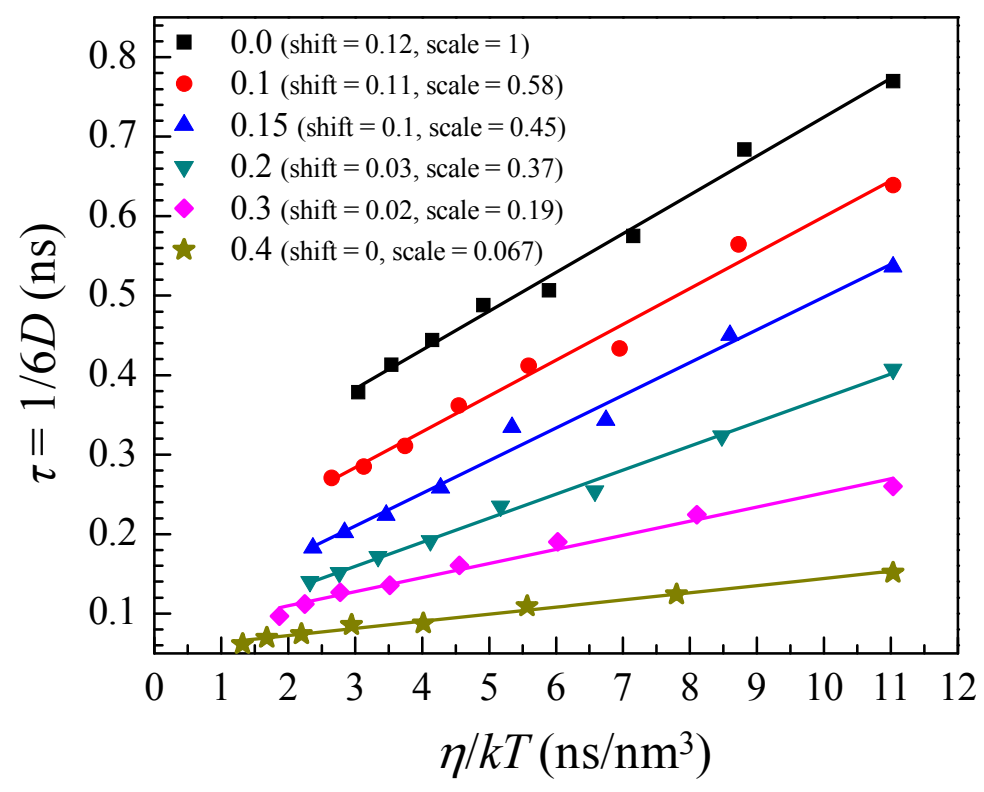

figure 5 


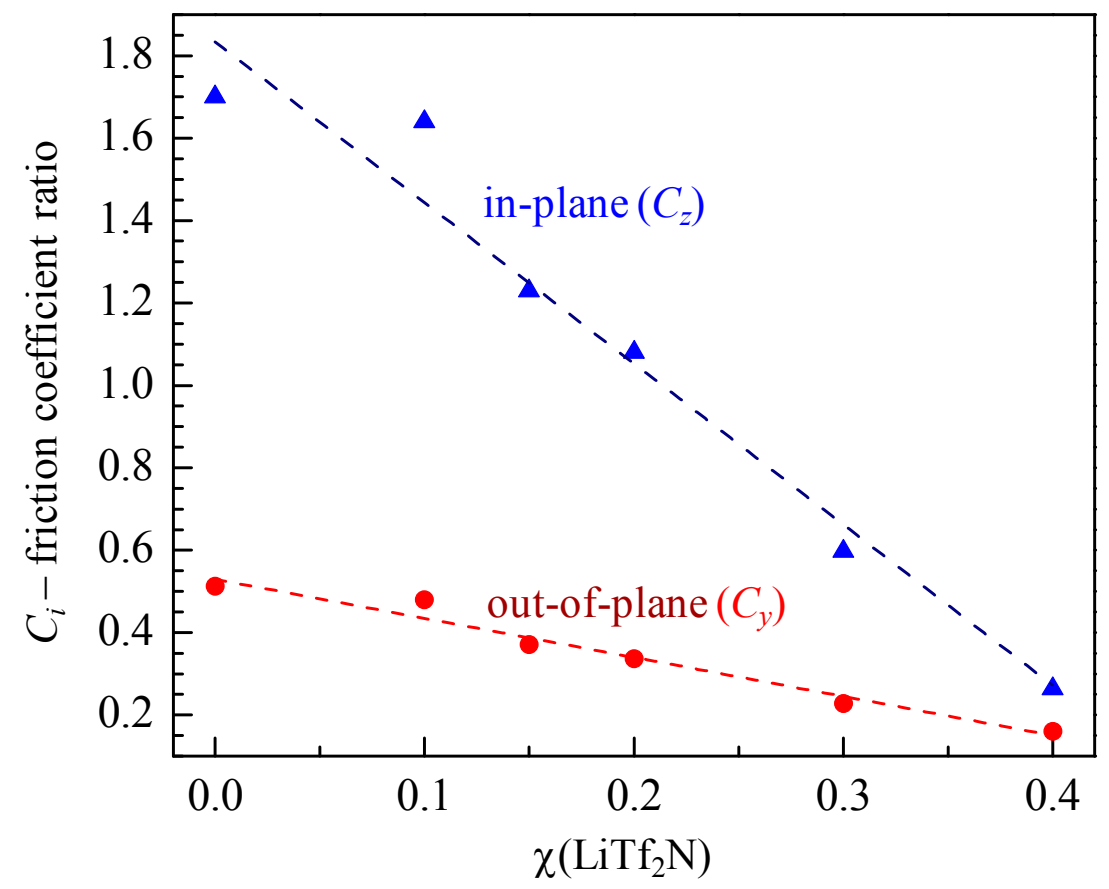

figure 6

29

30

31

32

33

34

35

36

37

38

39

40

41

42

43

44

45

46

47

48

49

50

51

52

53

54

55

56

57

58

59

60

61

62 


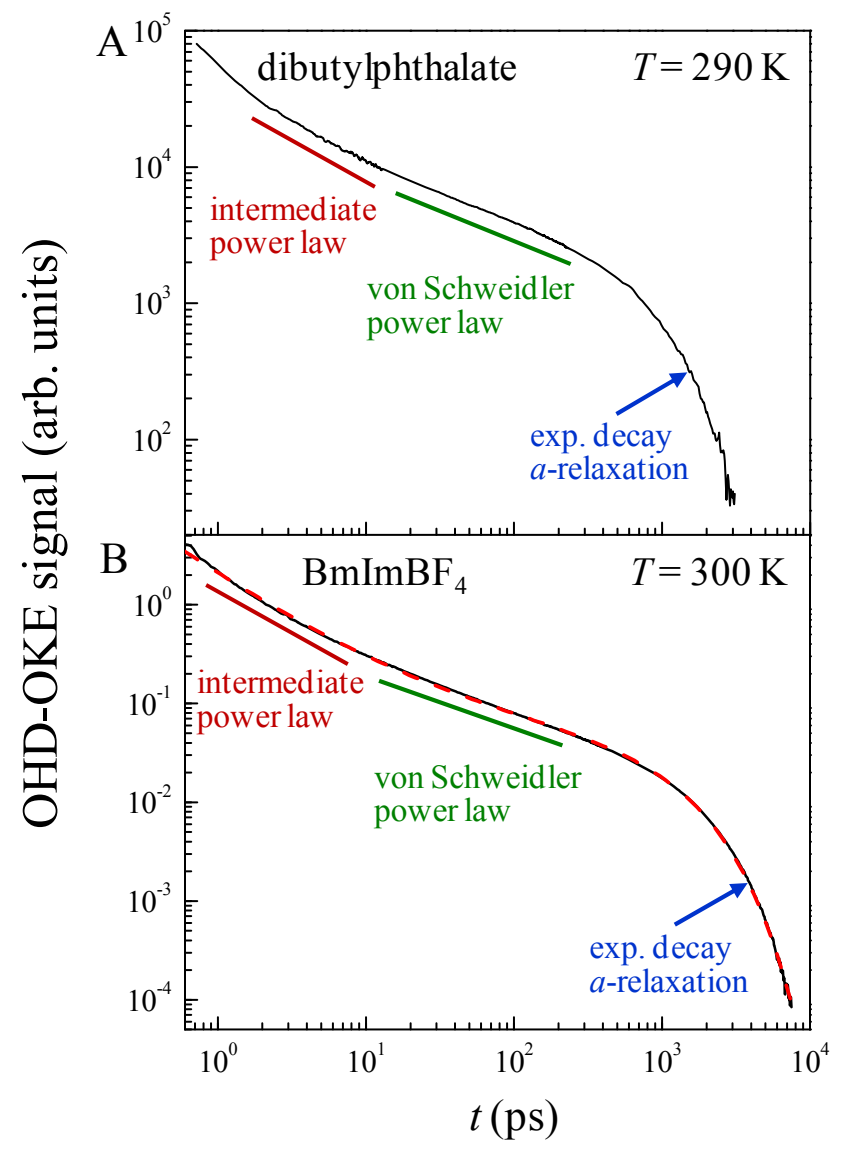

figure 7 


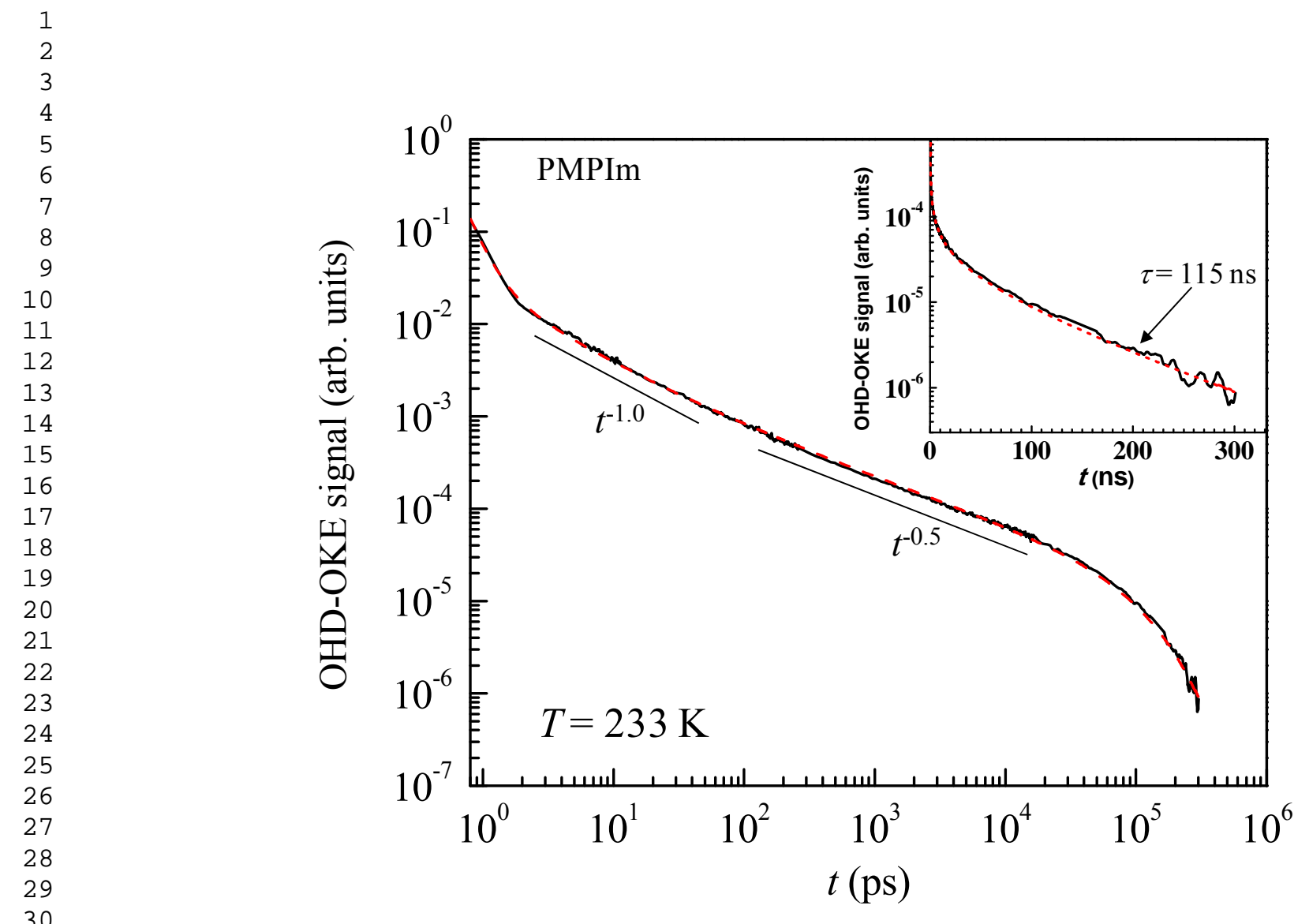

figure 8 


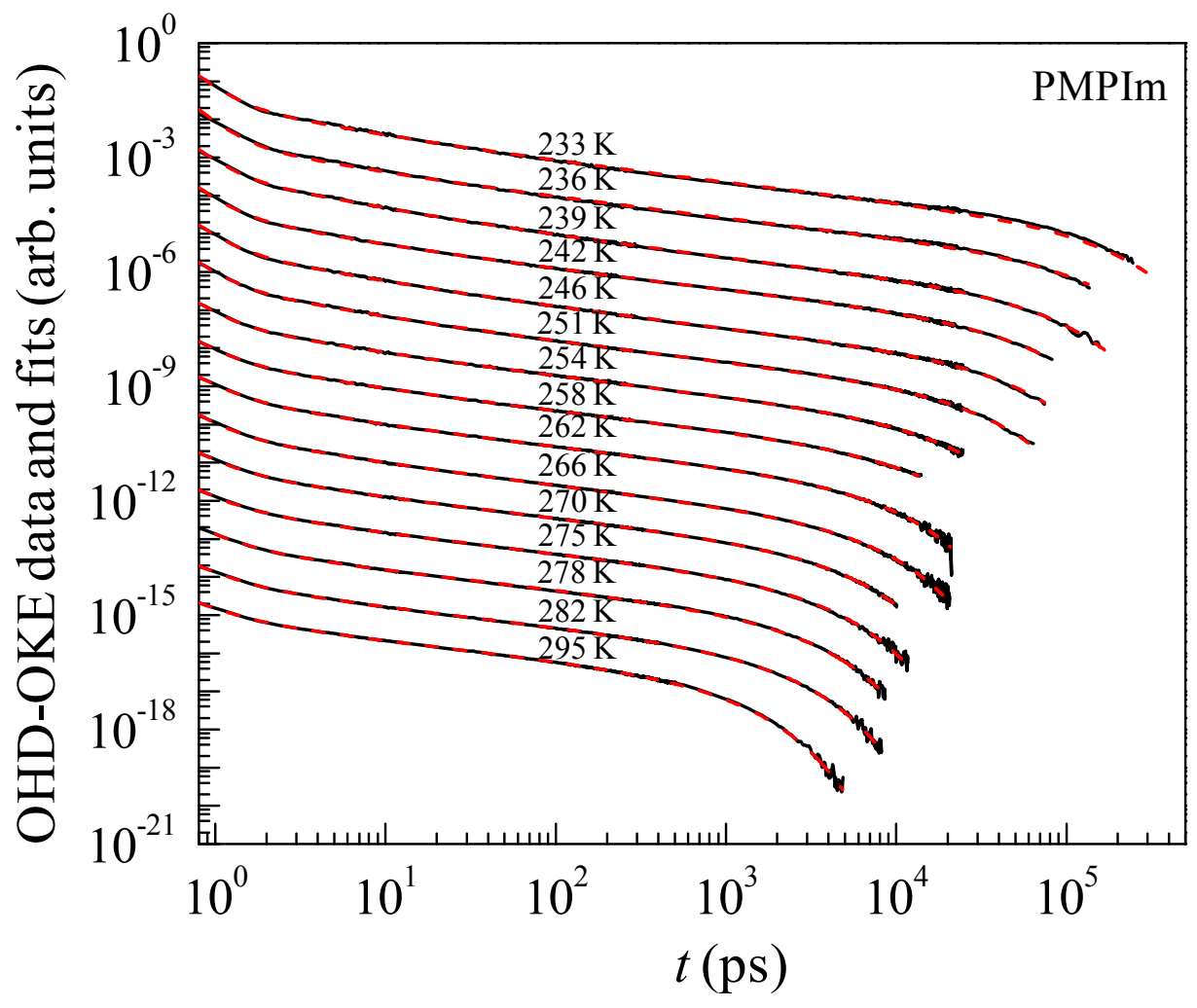

figure 9 


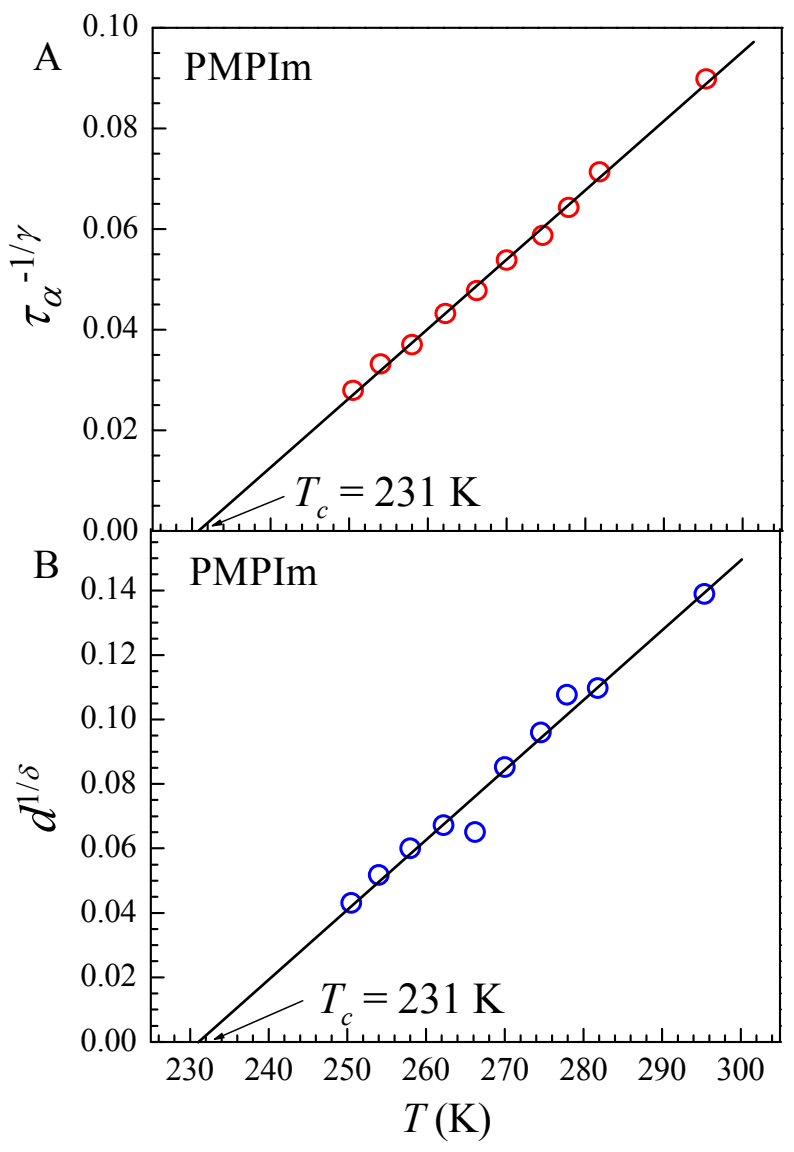

figure 10 


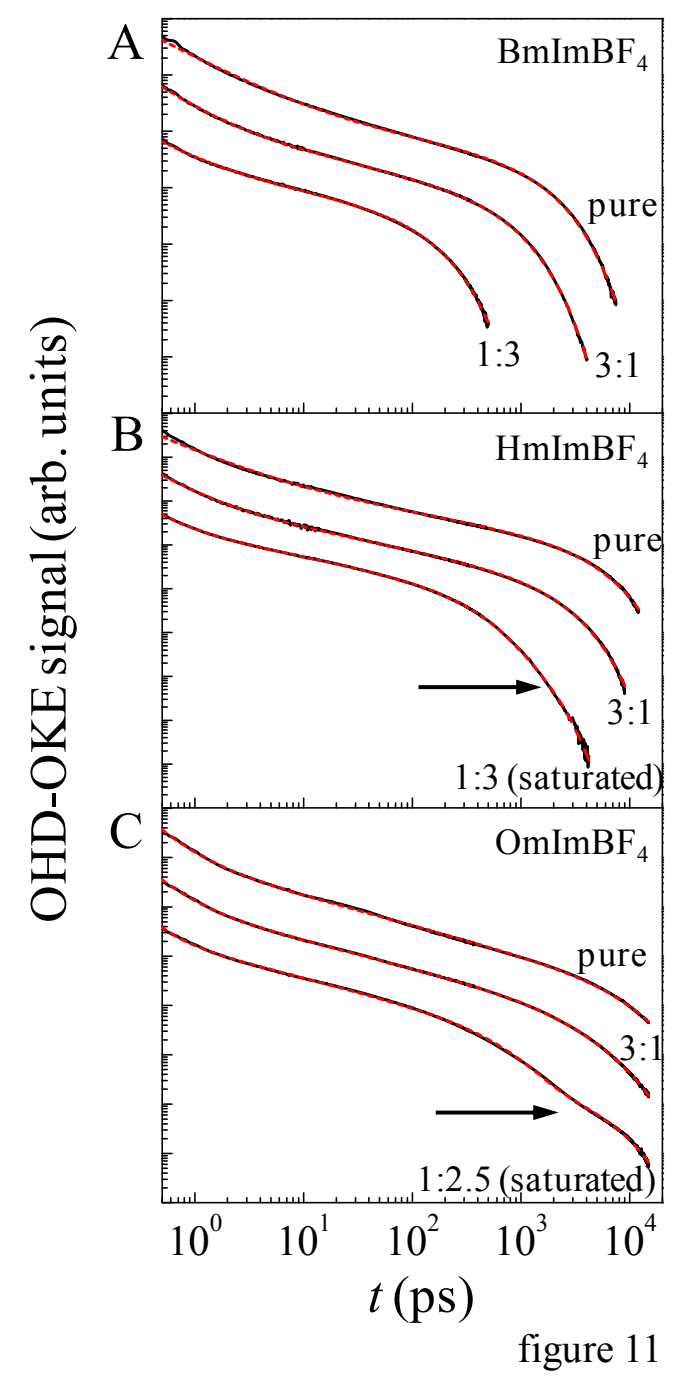

39

40 


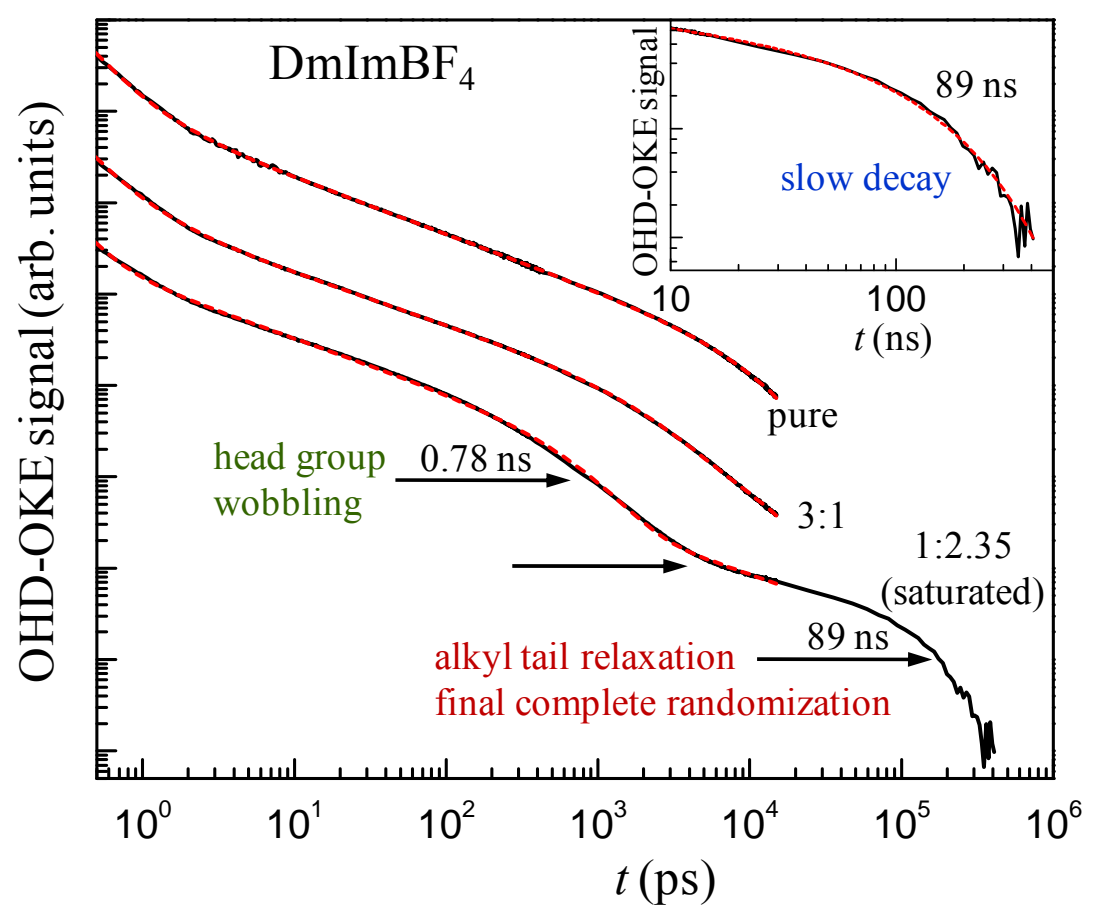

figure 12 

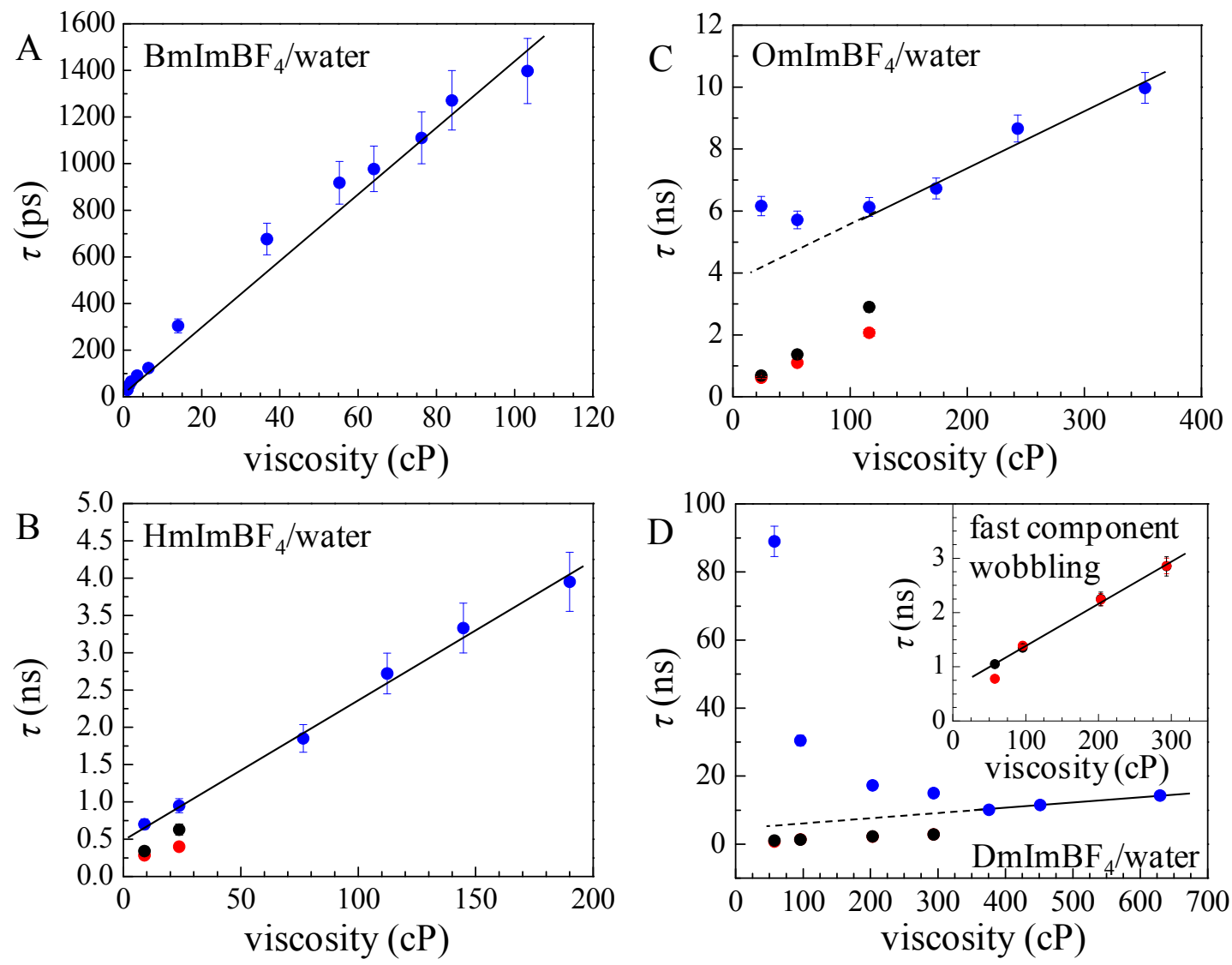

figure 13 


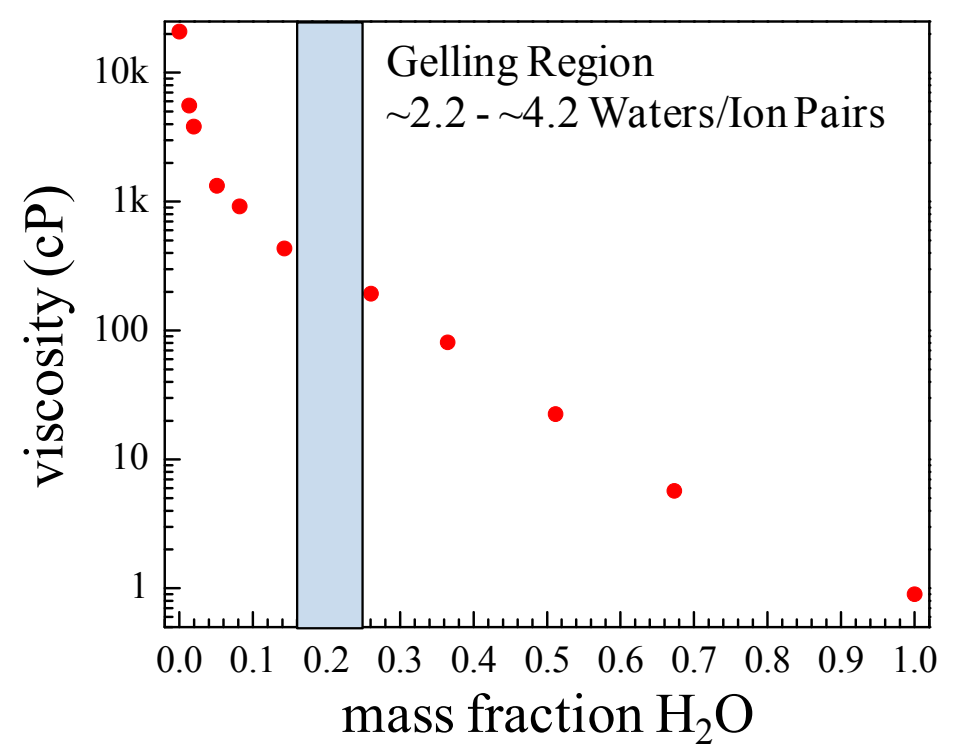

figure 14

28

29

30

31

32

33

34

35

36

37

38

39

40

41

42

43

44

45

46

47

48

49

50

51

52

53

54

55

56

57

58

59

60

61

62

63 


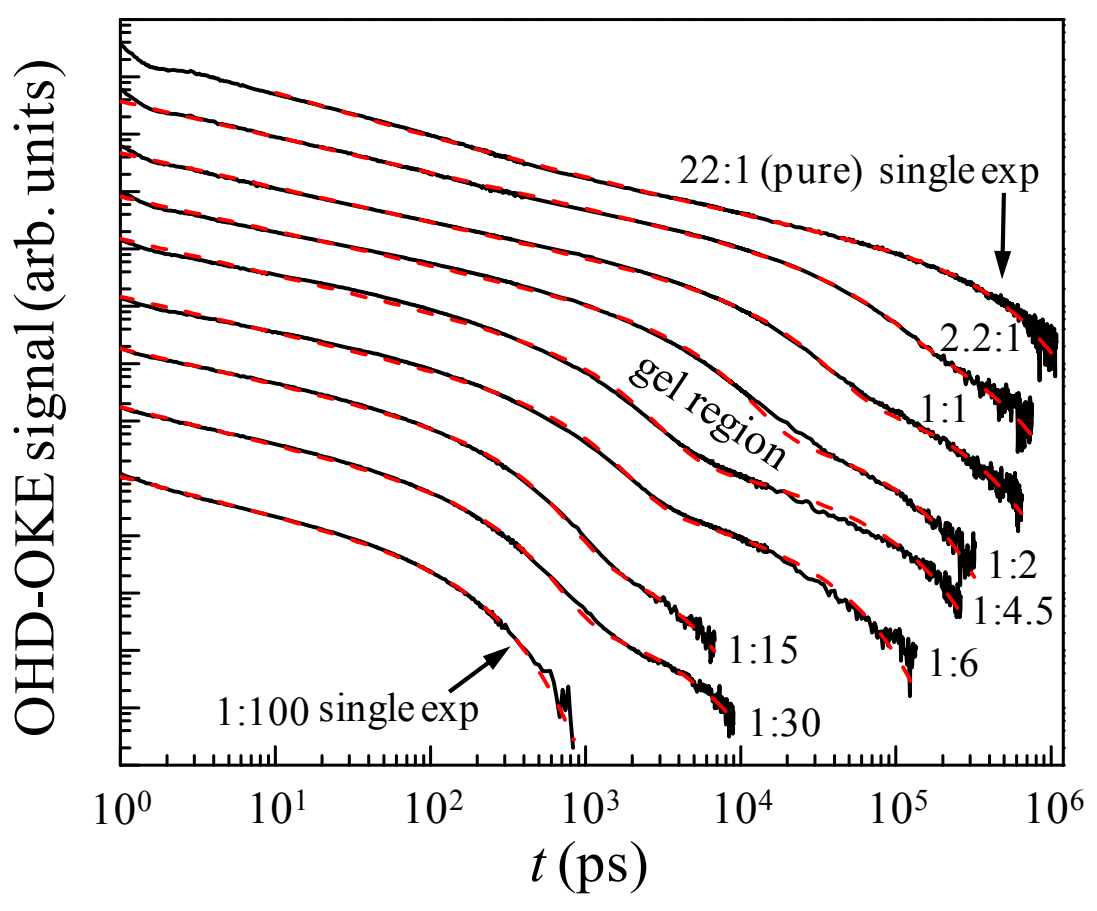

figure 15 


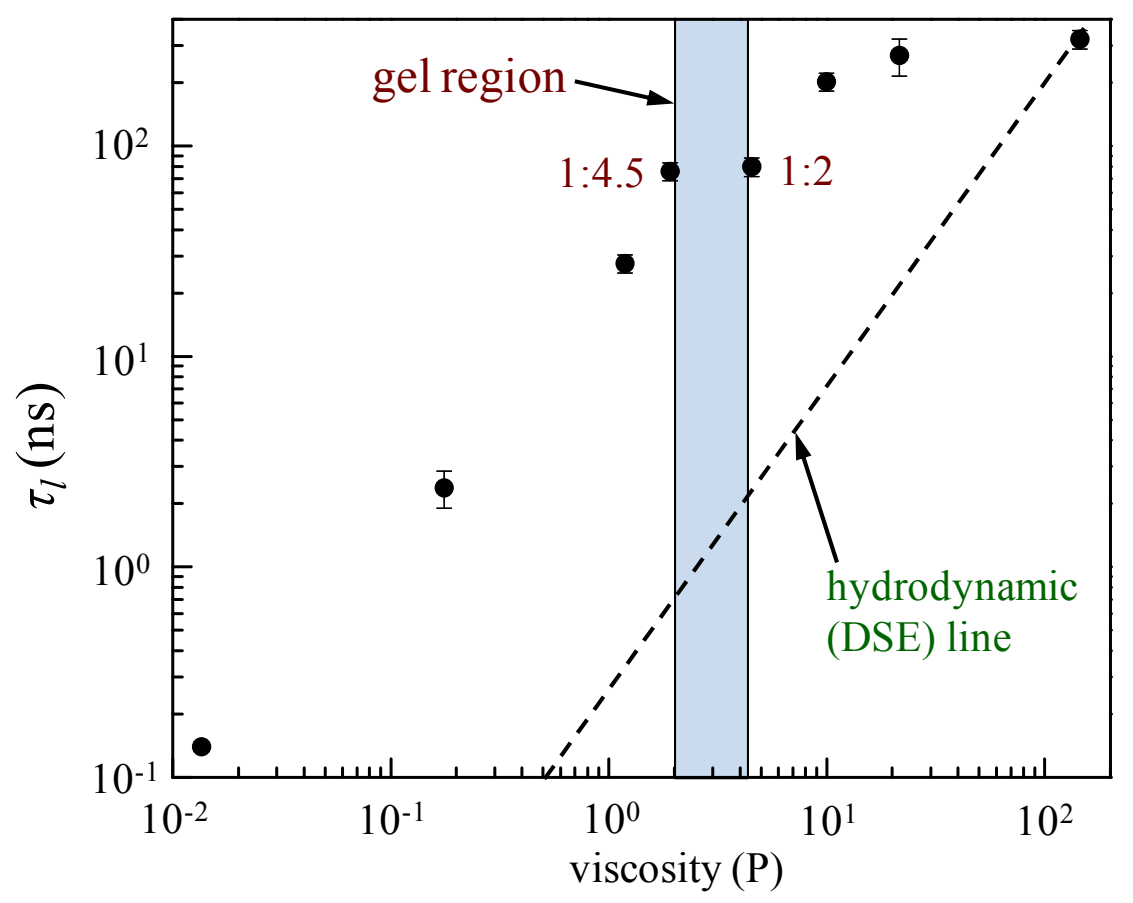

figure 16 


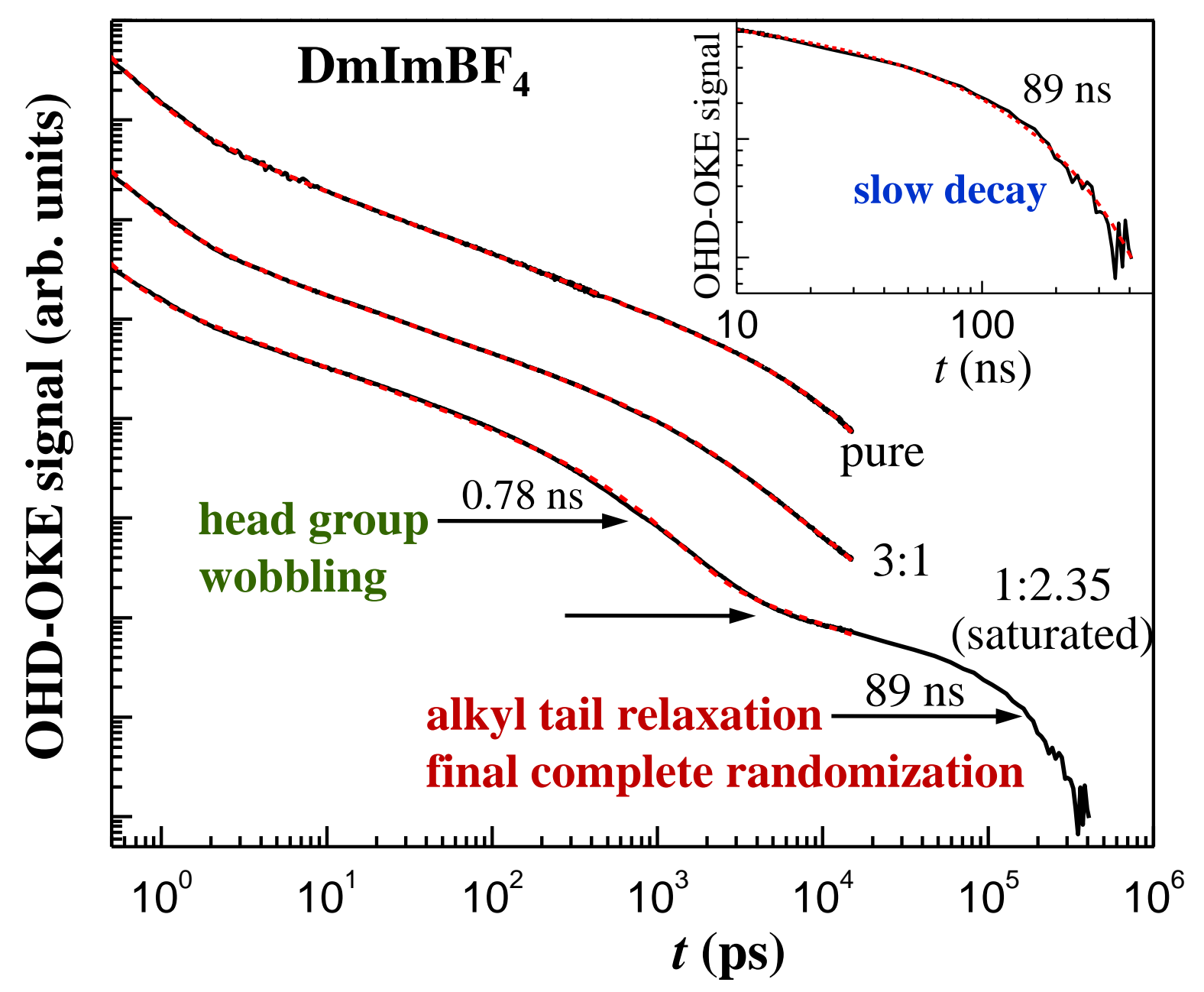

